Article

\title{
A Theoretical Study on Performance and Design Optimization of Linear Dielectric Compound Parabolic Concentrating Photovoltaic Systems
}

\author{
Guihua Li, Jingjing Tang and Runsheng Tang *(i) \\ Education Ministry Key Laboratory of Advanced Technology and Preparation for Renewable Energy Materials, \\ Yunnan Normal University, Kunming 650500, China; guihuali688@163.com (G.L.); jjtang166@126.com (J.T.) \\ * Correspondence: kingtang01@126.com
}

Received: 28 August 2018; Accepted: 13 September 2018; Published: 15 September 2018

\begin{abstract}
To investigate solar leakage and effects of the geometry of linear dielectric compound parabolic concentrator with a restricted exit angle (DCPC $-\theta_{a} / \theta_{e}$ ) on the performance of DCPC $-\theta_{a} / \theta_{e}$ -based photovoltaic systems $\left(\mathrm{DCPV}-\theta_{a} / \theta_{e}\right)$, a three-dimensional radiation transfer model based on solar geometry and vector algebra is suggested. Analysis shows that the annual radiation loss due to leakage is sensitive to the geometry of DCPCs and tilt-angle adjustment strategy, and the optimal $\theta_{e, o p t}$ for minimizing annual leakage is the one that makes the incidence angle of solar rays on the plane wall equal to the critical incidence angle for total internal reflection at solar-noon in solstices and days when tilt-angle adjustment from site latitude is made for DCPV with the aperture's tilt-angle being yearly fixed, and adjusted two and four times, respectively. It is found that annual radiation leakage is considerable small, for DCPVs with $\theta_{e}<\theta_{e, o p t}$, almost all leaked radiation comes from sky diffuse radiation, whereas for $\theta_{e}=90^{\circ}$, most of leakage is attributed to direct sunlight. As compared to similar non-concentrating solar cells, more radiation arrives annually on solar cells of DCPV- $\theta_{a} / \theta_{e}$ at small angles thanks to refraction of radiation on the aperture, hence, under same operation conditions, the annual average photovoltaic efficiency of solar cells for concentrated radiation is even higher. Analysis also shows that the power increase of DCPVs, being much less than the geometric concentration of DCPCs $\left(C_{t}\right)$, is mainly attributable to optical loss due to absorption of solar rays on the way to the solar cells, and the power loss due to leakage of radiation is not significant. From the point of annual electricity generation, for full DCPVs with a given $\theta_{a}, \mathrm{DCPV}-\theta_{a} / 90$ are favorable, and for truncated DCPVs with given $\theta_{a}$ and $C_{t}$, DCPVs with $\theta_{e}<90$ are favorable; whereas from the point of contribution per unit volume of dielectric to the annual electricity generation, the situation is reversed.
\end{abstract}

Keywords: linear dielectric compound parabolic concentrator; three-dimensional radiation transfer; solar leakage; performance investigation

\section{Introduction}

Concerns about energy and the environment are daily ubiquitous topics worldwide, however at present the rising energy demand is in opposition to environmental protection as most of energy comes from fossil fuels. Therefore, aggressive energy production reforms are necessary, and one of best solutions is the use of renewable energy. Among renewable energy sources, electricity generated by photovoltaic techniques has attracted much attention, but the application is still limited due to its high cost in comparison to conventional electricity generation. The cost of electricity from a photovoltaic system can be reduced by tracking the Sun [1,2] or concentrating solar radiation onto solar cells [3,4]. In practical applications, photovoltaic system equipped with continuous Sun-tracking devices often 
suffer from mechanical failures, and the use of cheap stationary optics for concentrating solar radiation on solar cells are commonly considered as one of best solutions to reduce the cost of electricity from PV systems. Low concentrator photovoltaic systems (CPVs), sharing advantages of simpler structures, easy control and no need for Sun-tracking, were widely tested in recent years [3]. CPVs also offer broad prospects for the integration of PV systems into buildings. Mallick and Eames performed a series of experimental studies on asymmetric compound parabolic concentrator (CPC)-based photovoltaic systems for building façade integration of PV systems [5-7], and found that, compared with similar non-concentrating PV panels, the use of an asymmetric reflective CPC $(2.01 \times)$ increased the maximum power point of photovoltaic modules by $62 \%$, and can reduce the cost of electricity from PV systems by up to $40 \%$. Brogren et al. [8] tested a CPC (3×)-based $\mathrm{Cu}(\mathrm{In}, \mathrm{Ga}) \mathrm{Se}_{2}$ PV module, and a 1.9-fold power increase was measured. Yousef et al. [9] tested a CPV $(2.4 \times)$ with a polished stainless-steel reflector and found that, as compared to similar solar panels, the electricity from the CPV with and without cooling of solar cells was increased by $52 \%$ and $33 \%$, respectively. These works indicated that reflective CPCs can increase the power output, but the increase factor was much less than the geometric concentration due to optical losses resulting from imperfect reflection of the solar rays on their way to the solar cells [10] and electrical losses resulting from higher cell temperatures, inhomogeneous irradiation and increased incidence angles on the solar cells [11-13]. The inhomogeneous irradiation on solar cells usually creates hotspots, leading to current mismatch and tending to reduce their power output and durability [14]. To overcome the shortcoming of uneven irradiation on solar cells, Hatwaambo et al. $[15,16]$ tested a CPC with rolling marks on reflectors and found that it can increase the fill factor of CPVs, but it leads to a decrease in collectible radiation due to diffuse reflection [17]. To simplify the manufacture and make the solar flux on solar cells more uniform, Tang et al. proposed the compound plane concentrators (CPC-A) concept as an alternative to CPCs, and found that an optimized CPC-A annually collects almost identical radiation to that of a CPC [18]. Experimental studies by Baig et al. showed that inhomogeneous irradiation resulted in about $0.5 \%$ drop of solar cell efficiency of a linear dielectric CPC-based PV system [19], and Yu experimentally found that the power output of solar cells is highly sensitive to the incidence angle of solar rays on the cells $\left(\theta_{\text {in }}\right)$ when $\theta_{\text {in }}>60^{\circ}$ but weakly so to the solar flux distribution [20]. An experimental study by Bahaidarah et al. showed that a glazed PV/T system would greatly reduce the power output and the unglazed system was advisable for greater electricity output [21]. A comparative experiment by Bahaidarah et al. showed that, as compared to similar PV panels, using polished stainless steel as the reflectors of CPC $(2.3 \times)$ increased the power output by $39 \%$ and $23 \%$ in the cases of cooling and non-cooling, respectively [22]. These results demonstrated that cooling the solar cells of CPVs is required to enhance the photovoltaic performance of CPVs and the heat can be extracted for other applications [23-25]. To reduce optical losses due to imperfect reflections, a lens-walled CPC was proposed by Su and Li et al. and their analysis showed that it shares higher optical efficiency, wider acceptance angle and more homogeneous irradiation in comparison to reflective CPCs [26,27]. To enhance solar absorption, CPCs with a restricted exit angle (CPC- $\left.\theta_{a} / \theta_{e}\right)$ were first suggested by Rabl and Winston [28]. A theoretical study by $\mathrm{Yu}$ et al. showed that the use of $\mathrm{CPC}-\theta_{a} / \theta_{e}$ makes more radiation arrive on solar cells at small angles, thus improving the photovoltaic performance of CPVs [29]. Comparative experiments by Yu et al. showed that the power output from a CPV-20/65 setup was slightly higher as compared to a CPV-20/90 one [20].

As compared to reflective CPCs, dielectric internally reflecting compound parabolic concentrators (DCPCs) share advantages of wider acceptance angles and higher optical efficiency. The earlier work of Winston indicated that, for a given source, the use of dielectric with a refractive index $n$ increases the geometric concentration of linear CPCs by a factor of $n$ [30]. Previous work of the authors shows that, given the minimum daily hours for concentrating direct radiation, the use of dielectric increase the maximum geometric concentration of DCPC by a factor larger than $n$ [31]. Experiments by Muhammad-Sukki et al. demonstrated that, as compared to a similar PV panel, a DCPC $(4.9 \times)$-based photovoltaic system increased the maximum power point by a factor of 4.2 [32]. Ray tracing analysis 
by Sellami and Mallick showed that a crossed DCPC $(3.6 \times)$ has a maximum optical efficiency of 95\% [33]. Baig et al. tested a building-integrated concentrating photovoltaic (BICPV) system $(6 \times)$, and a maximum temperature of $319 \mathrm{~K}$ was observed on the solar cell surface under normal incidence, and a 3.4 power increase ratio was measured [34]. A study by Zacharopoulos indicated that a linear DCPC, made of low-iron glass, had an optical efficiency over $90 \%$ for a wide range of incidence angles and over $40 \%$ even for incidence angles outside its acceptance angle, furthermore, most of radiation arrived on solar cells at the angle of less than $40^{\circ}$ [35]. Brunotte and Hen tested a crossed DCPC-23.45/60 as the second stage of a primary parabolic trough for use with solar cells, and an optical efficiency of up to $89 \%$ and $80 \%$ was measured [36,37]. Ray tracing analysis and experiments by Pei et al. indicated that a fraction of the radiation incident on the walls of a DCPC can't undergo internal reflection, even within the acceptance angle [38]. Ray tracing analysis by Baig et al. indicated that metalizing the external walls of a linear DCPC $(2.8 \times)$-based PV system increased the average power output by $16 \%$ [19], and experiments showed that adding the reflective film only resulted in about $0.5 \%$ drop of the maximum power output due to the effect of non-uniformity [39]. However, the use of reflective film on the external walls of DCPC would result in an extra optical loss due to imperfect reflections. To avoid solar leakage, a DCPC with a restricted exit angle (DCPC- $\theta_{a} / \theta_{e}$ ) is advisable [31]. However, for DCPC- $\theta_{a} / \theta_{e}$, the radiation irradiating on upper parabolic walls at angle larger than $\theta_{a}$ would redirect to the opposite plane walls and then probably leak to air.

Linear CPCs are usually mounted towards to the equator and oriented in the east-west direction for efficient radiation collection, and a two-dimensional radiation transfer model can reasonably predict the optical performance of reflective CPCs $[13,40,41]$ and is thus widely employed [42-44]. However, for linear DCPCs, the projected angles of incident and refractive rays at the air-dielectric aperture are usually not subject to the Snell's law relationship [31,45], furthermore, whether solar rays incident on internal walls of DCPCs are totally internally reflected onto the absorber is uniquely determined by their real incidence angle, and absorption of solar rays on way to the absorber is dependent on the path length. This means that any 2-D radiation transfer model can't reasonably predict the optical performance of a DCPC. Three-dimensional ray trace analysis can be conveniently used to investigate the angular dependence of DCPCs [35], but not practical for investigating the long-term performance of DCPCs due to a large number of ray-tracing analysis necessary to find transversal and longitudinal angular dependences of the optical efficiency at any time on all days of a year. In addition, DCPC optical loss due to leakage is almost impossible to measure directly or quantitatively estimate by ray tracing analysis. Hence, it is necessary to develop a mathematical procedure for investigating the solar leakage and performance of DCPCs based on three-dimensional radiation transfer within the DCPC. In the previous work of authors, the DCPC design is addressed [31], but the effects of DCPC geometry on the performance of DCPC-based photovoltaic systems were not investigated. In this work, a three-dimensional radiation transfer model drawing inspiration from previous works of authors [13,31] is suggested, and optical loss due to leakage from walls and the effects of the DCPC geometry on the DCPV performance are studied.

\section{Three-Dimensional Radiation Transfer Model}

\subsection{Equation of Linear DCPC's Walls}

The wall of a DCPC $-\theta_{a} / \theta_{e}$ consists of an upper parabola and lower plane. The right parabolic wall in the coordinate system shown in Figure 1 is expressed by [46]:

$$
\left\{\begin{array}{c}
z=\frac{a\left(\sin \theta_{e}+\sin \theta_{a}\right) \sin \varphi}{1-\cos \left(\varphi+\theta_{a}\right)}-0.5 a \\
x=\frac{a\left(\sin \theta_{e}+\sin \theta_{a}\right) \cos \varphi}{1-\cos \left(\varphi+\theta_{a}\right)}
\end{array} \quad\left(\theta_{t} \leq \varphi \leq \theta_{e}\right)\right.
$$


where $a$ is the width of DCPCs' base to which solar cells are attached, $\varphi$ is the polar angle; the $\theta_{e}$ is the maximum exit angle of DCPC $-\theta_{a} / \theta_{e}$ for refractive radiation within its acceptance angle $\left(\theta_{a}\right)$, and $\theta_{e}$ is the edge-ray angle after truncation. The plane wall of DCPC $-\theta_{a} / \theta_{e}$ is expressed by:

$$
x=c \tan \gamma_{p l}(z-0.5 a) \quad\left(0.5 a \leq z \leq z_{D}\right)
$$

where $\gamma_{p l}=0.5\left(\theta_{e}-\theta_{a}\right)$, is the tilt angle of plane wall relative to $\mathrm{x}$-axis [29]. The cross-section area of linear DCPCs is given by:

$$
A_{c}=\left(z_{D}+0.5 a\right) x_{D}+2 \int_{\theta_{t}}^{\theta_{e}} z d x
$$

where $x_{D}$ and $z_{D}$ are $\mathrm{x}$ - and $\mathrm{z}$-coordinates of lower point $\mathrm{D}$ of the parabolic wall, respectively.

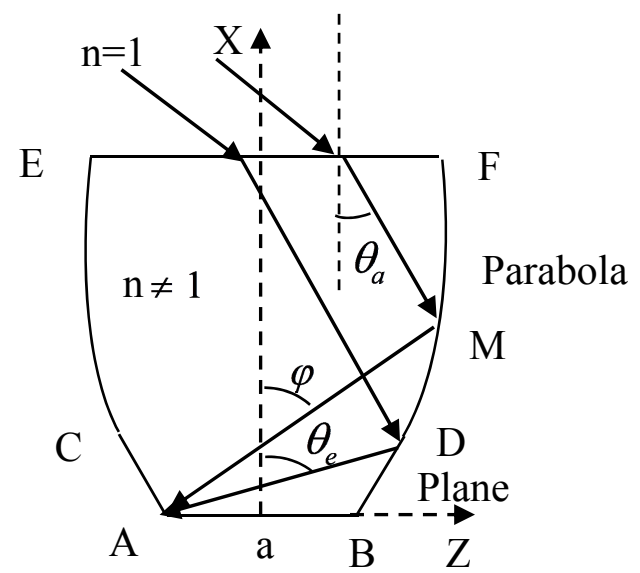

Figure 1. Geometry of DCPC $-\theta_{a} / \theta_{e}$.

\subsection{Vectors of Incident and Refractive Rays}

The linear DCPCs investigated in this work are mounted in an east-west direction and tilted at $\beta$ from the horizon. For convenience of analysis, a coordinate system with $x$-axis normal to the aperture, $y$-axis pointing to the east and z-axis pointing to northern sky dome is employed (see Figure 2).

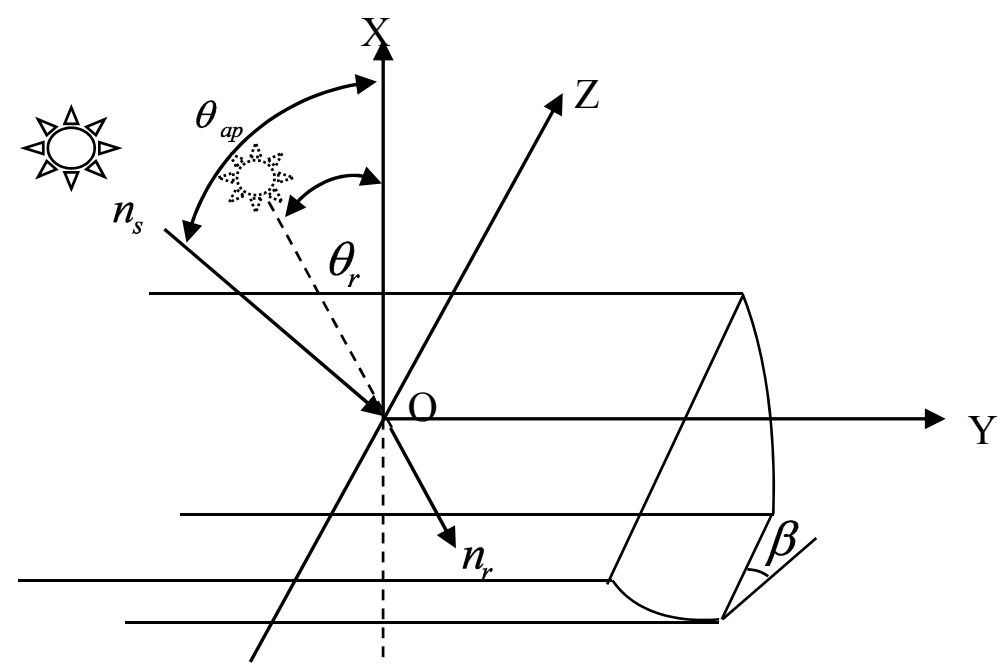

Figure 2. Vectors of incident and refractive rays.

The unit vector of incident ray from Earth to the Sun is expressed by [2,31]:

$$
n_{s}=\left(n_{x}, n_{y}, n_{z}\right)
$$


where:

$$
\left\{\begin{array}{l}
n_{x}=\cos \delta \cos \omega \cos (\lambda-\beta)+\sin \delta \sin (\lambda-\beta) \\
n_{y}=-\cos \delta \sin \omega \\
n_{z}=-\cos \delta \cos \omega \sin (\lambda-\beta)+\sin \delta \cos (\lambda-\beta)
\end{array}\right.
$$

where $\lambda$ is the site latitude, $\omega$ the solar hour angle, and $\delta$ the declination of the Sun. The unit vector of refractive rays from Earth to the "virtual Sun" seen within the dielectric is expressed by [31]:

$$
n_{r}=\left(n_{x 1}, n_{y 1}, n_{z 1}\right)
$$

where:

$$
\left\{\begin{array}{l}
n_{x 1}=\cos \theta_{r}=\sqrt{1-\left(1-n_{x}^{2}\right) / n^{2}} \\
n_{y 1}=n_{y} / n \\
n_{z 1}=n_{z} / n
\end{array}\right.
$$

The projected angle of a refractive ray on the cross-section of DCPCs (see Figure 3) is given by:

$$
\tan \theta_{p}=\left|n_{z 1} / n_{x 1}\right|
$$

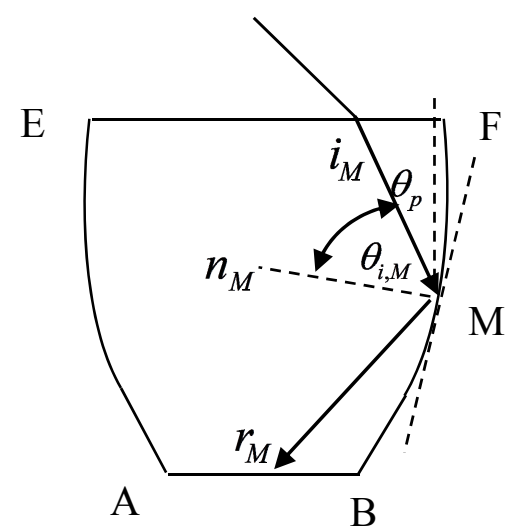

Figure 3. Incident angle of solar rays on the internal wall of DCPCs.

The incidence angle of solar rays at point M of the right parabolic wall of DCPCs (see Figure 3) is given by:

$$
\cos \theta_{i, M}=n_{r} \cdot n_{M}
$$

where $n_{M}=\left(\sin \gamma_{M}, 0,-\cos \gamma_{M}\right)$ is the unit vector of normal to parabolic wall at point $M$, and the tilt-angle of the line tangent to parabolic wall at point $M$ relative to $x$-axis is obtained by Equation (1) as:

$$
\tan \gamma_{M}=d z / d x=\left(\cos \theta_{a}-\cos \varphi_{M}\right) /\left(\sin \theta_{a}+\sin \varphi_{M}\right)
$$

Substituting Equation (6) into Equation (9) one obtains:

$$
\cos \theta_{i, M}=n_{x 1} \sin \gamma_{M}-n_{z 1} \cos \gamma_{M}
$$

For symmetric DCPCs, the optical and photovoltaic performance for radiation $n_{s}=\left(n_{x}, n_{y}, \pm n_{z}\right)$ are identical. Hence, to simplify our analysis, in this work it is assumed that the radiation is always incident towards onto right wall of DCPVs, i.e., $n_{s}=\left(n_{x}, n_{y},-\left|n_{z}\right|\right)$, therefore, radiation irradiating on the left wall will be totally internally reflected onto solar cells for $n>1.4$ as indicated in the previous work of the authors [31]. 


\subsection{Transmittance of Solar Rays at Air-Dielectric Aperture}

As solar rays are incident on the aperture of DCPVs, a fraction of radiation is directly reflected back to air, and the reflectivity for non-polarized and slightly polarized sunlight is subjected to Fresnel's Law as [40]:

$$
\rho_{a p}=0.5\left[\frac{\tan ^{2}\left(\theta_{a p}-\theta_{r}\right)}{\tan ^{2}\left(\theta_{a p}+\theta_{r}\right)}+\frac{\sin ^{2}\left(\theta_{a p}-\theta_{r}\right)}{\sin ^{2}\left(\theta_{a p}+\theta_{r}\right)}\right]
$$

where $\theta_{a p}$, the incident angle of solar ray on the aperture, is given by $\cos \theta_{a p}=n_{x}$. For $\theta_{a p}=0$, $\rho_{a p}=0.5(n-1)^{2} /(n+1)^{2}$. The transmittance of solar rays at the aperture of DCPVs is given by $\tau=1-\rho_{\text {ap }}$.

\subsection{Optical and Photovoltaic Performance of Linear DCPVs}

For DCPV- $\theta_{a} / \theta_{e}$, all refractive radiation within $\theta_{a}$ arrives on solar cells at angle $\left(\theta_{i n}\right)$ less than $\theta_{e}$, whereas for radiation incident at $\theta_{p}>\theta_{a}$, a fraction of radiation incident on plane wall and upper parabolic wall arrives on solar cells at $\theta_{i n}>\theta_{e}[20,46]$. Therefore, the radiation on solar cells of DCPVs at any time of a day includes six components: radiation directly irradiating on solar cells $\left(I_{1}\right)$, incident on right/left plane wall and arriving on solar cells $\left(I_{2} / I_{3}\right)$, incident on right/left parabolic wall and arriving on solar cells $\left(I_{4} / I_{5}\right)$, incident on right upper parabola at $\theta_{p}>\theta_{a}$ and arriving on solar cells $\left(I_{6}\right)$. Therefore, the optical efficiency of DCPVs is given by:

$$
f=\left(I_{1}+I_{2}+I_{3}+I_{4}+I_{5}+I_{6}\right) / I_{a p}=f_{1}+f_{2}+f_{3}+f_{4}+f_{5}+f_{6}
$$

where $I_{a p}$ is the radiation on the aperture; $f_{1}, f_{2}, f_{3}, f_{4}, f_{5}, f_{6}$ are the energy fraction of radiation on solar cells contributed by $I_{1}, I_{2}, I_{3}, I_{4}, I_{5}$ and $I_{6}$, respectively. Similarly, the photovoltaic efficiency of DCPVs is expressed by:

$$
\eta=\left(P_{1}+P_{2}+P_{3}+P_{4}+P_{5}+P_{6}\right) / I_{a p}=\eta_{1}+\eta_{2}+\eta_{3}+\eta_{4}+\eta_{5}+\eta_{6}
$$

where $P_{i}(i=1,2,3,4,5,6)$ is the power generation contributed by $I_{i}$, and the $\eta_{i}$ is the photovoltaic efficiency of DCPVs contributed by $P_{i}$.

\subsubsection{Calculation of $f_{1}$ and $\eta_{1}$}

As shown in Figure 4, the energy fraction of radiation directly irradiating on solar cells is given by:

$$
f_{1}=\tau \tau_{a, 1} \Delta z_{1} / a C_{t}
$$

where $\Delta z_{1} / a C_{t}$ is the fraction of radiation directly irradiating on solar cells and given by [13]:

$$
\Delta z_{1} / a C_{t}= \begin{cases}1 / C_{t} & \left(\theta_{p} \leq \gamma_{f}\right) \\ 0.5\left(1+C_{t}\right)\left(1-\tan \theta_{p} / \tan \theta_{t}\right) / C_{t} & \left(\gamma_{f}<\theta_{p} \leq \theta_{t}\right) \\ 0 & \text { else }\end{cases}
$$

where $\gamma_{f}$ is subjected to $\tan \gamma_{f}=\left(C_{t}-1\right) \tan \theta_{t} /\left(C_{t}+1\right), \tau_{a, 1}=\exp \left(-k L_{1}\right)$ is the transmittance of solar ray on way from the aperture to solar cells, the $k$ is the extinction coefficient of dielectric, and $L_{1}$ is the path length of solar ray from the aperture to solar cells and given by:

$$
L_{1}=h / \cos \theta_{r}
$$

where $h=0.5 a\left(1+C_{t}\right) / \tan \theta_{t}$ is the height of DCPVs. The photovoltaic efficiency of DCPVs due to the contribution of $P_{1}$ is given by:

$$
\eta_{1}=\tau \tau_{a, 1} \eta_{p v}\left(\theta_{i n, 1}\right) \Delta z_{1} / a C_{t}=f_{1} \eta_{p v}\left(\theta_{i n, 1}\right)
$$


where $\theta_{i n, 1}=\theta_{r}$ is the incident angle of solar rays on solar cells, $\eta_{p v}\left(\theta_{i n, 1}\right)$ is the photovoltaic efficiency of solar cells as a function of $\theta_{i n, 1}$. Under given operation conditions, the photovoltaic conversion efficiency of solar cells is mainly affected by cell temperature, solar incidence angle and solar flux distribution over cells $[19,20,47]$. In order to investigate effects of geometry of DCPVs on the photovoltaic performance, it is assumed that, DCPVs and similar PV panels operate at the same temperature, effects of solar flux distribution on $\eta_{p v}$ of DCPV with different geometry are identical, and angular dependence of $\eta_{p v}$ is subjected to the correlation suggested by $\mathrm{Yu}$ [20] as:

$$
\eta_{p v}= \begin{cases}15.5494+0.02325 \theta_{\text {in }}-0.00301 \theta_{\text {in }}^{2}+9.4685 \times 10^{-5} \theta_{\text {in }}^{3}-1.134 \times 10^{-6} \theta_{\text {in }}^{4} & \left(0 \leq \theta_{\text {in }} \leq 65^{\circ}\right) \\ 41.52-0.4782 \theta_{\text {in }} & \left(65^{\circ}<\theta_{\text {in }}<90^{\circ}\right)\end{cases}
$$

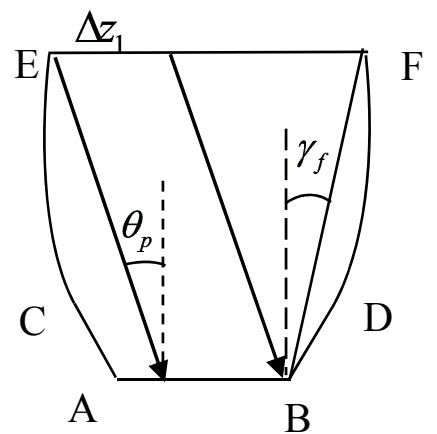

Figure 4. Fraction of radiation directly irradiating on solar cells.

\subsubsection{Calculation of $f_{2}$ and $\eta_{2}$}

For DCPVs, $\theta_{a}$ is usually small even for 1T-DCPVs [31], thus DCPV is commonly subjected to $\theta_{a}<2 \theta_{e}-0.5 \pi$, and for such DCPCs, radiation incident on the plane walls will arrive on solar cells after one reflection [29]. It is known from the imaging principle of plane mirrors that solar rays directing to the image of solar cells formed by the plane mirror will arrive solar cells after reflections [48]. As shown in Figure 5, $B A^{\prime}$ is the image of solar cells formed by right plane wall, therefore radiation incident on the entire plane wall (see Figure 5a), lower part BM (see in Figure 5b) and middle part MN (see Figure 5c) of the plane wall will be reflected onto solar cells for $\theta_{p} \leq \theta_{a}, \theta_{a}<\theta_{p} \leq \theta_{t}$ and $\theta_{t}<\theta_{p}<\theta_{p, c 1}$, respectively; whereas for $\theta_{p} \geq \phi_{a p}, B A^{\prime}$ is fully shaded by left wall, thus no radiation arrives on the solar cells (see Figure $5 \mathrm{~d}$ ). Therefore, one has:

$$
\begin{gathered}
f_{2}=\tau \tau_{a, 2} \Delta z_{2} \rho\left(\theta_{i, p l}\right) / a C_{t} \\
\eta_{2}=\tau \tau_{a, 2} \eta_{p v}\left(\theta_{i n, 2}\right) \Delta z_{2} \rho\left(\theta_{i, p l}\right) / a C_{t}=f_{2} \eta_{p v}\left(\theta_{i n, 2}\right)
\end{gathered}
$$

where $\theta_{i, p l}$ is the incident angle of solar rays on the plane wall and calculated based on Equation (11) by setting $\gamma_{M}=\gamma_{p l}$; and $\rho\left(\theta_{i, p l}\right)$, the reflectivity of solar rays on right plane wall, is 1 for $\theta_{i, p l} \geq \theta_{c}$, the critical incident angle for total internal reflection, otherwise calculated based on Fresnel's law as Equation (12). 

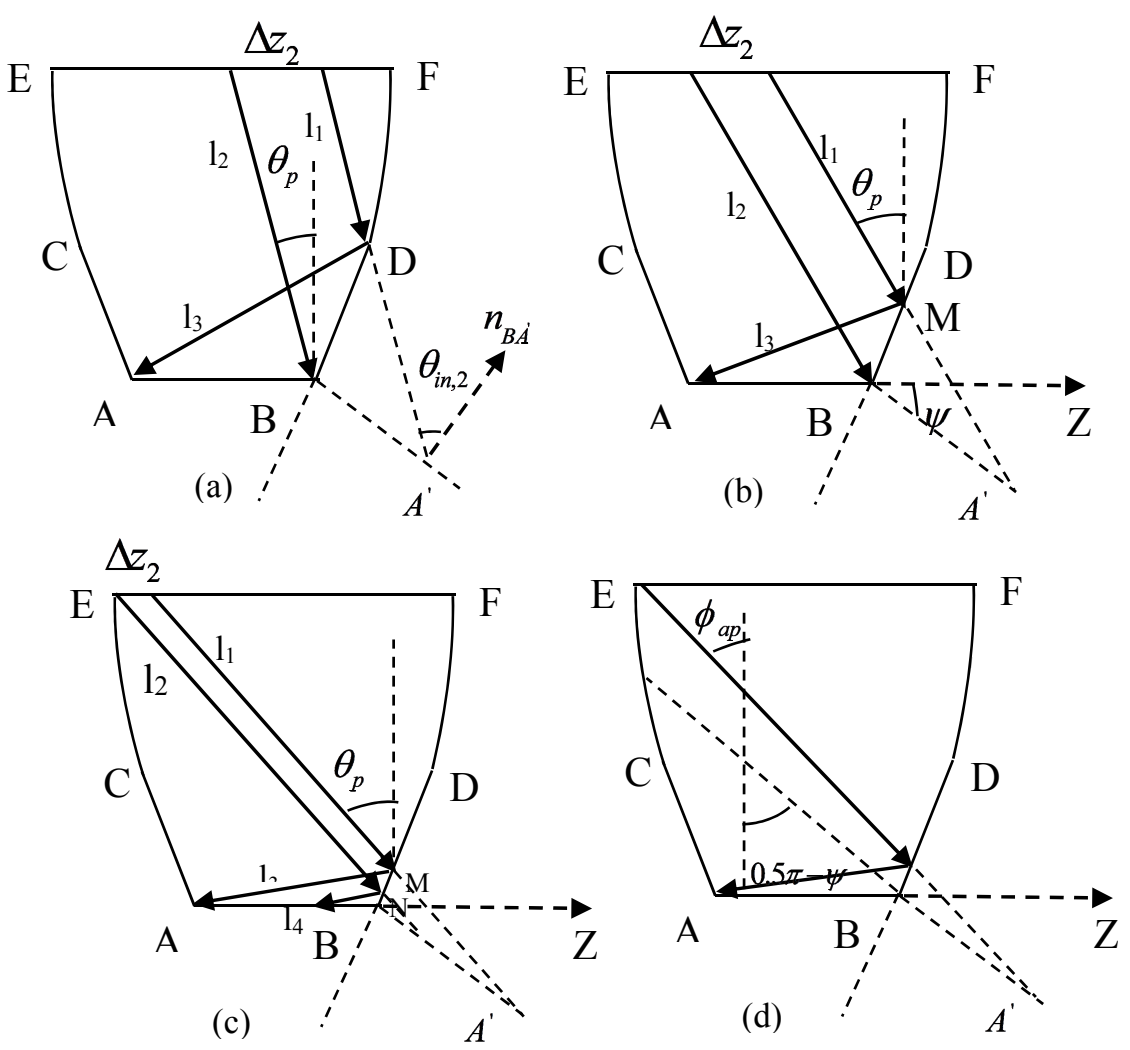

Figure 5. Fraction of refractive radiation incident on right plane wall and arriving on solar cells. (a): $\theta_{p} \leq \theta_{a},(\mathbf{b}): \theta_{a}<\theta_{p} \leq \theta_{t},(\mathbf{c}): \theta_{a}<\theta_{p} \leq \theta_{t} ;(\mathbf{d}) \theta_{p}>\operatorname{Min}\left(\phi_{a p}, 0.5 \pi-\psi\right)$.

$\Delta z_{2}$ in Equation (20) is given by $[13,29]$ :

$$
\Delta z_{2}= \begin{cases}z_{D}-0.5 a+x_{D} \tan \theta_{p} & \theta_{p} \leq \theta_{a} \\ a\left(\cos \psi-\sin \psi \tan \theta_{p}\right) & \theta_{a}<\theta_{p} \leq \theta_{t} \\ (h+a \sin \psi)\left(\tan \phi_{a p}-\tan \theta_{p}\right) & \theta_{t}<\theta_{p} \leq \theta_{p, c 1} \\ 0 & \theta_{p}>\theta_{p, c 1}\end{cases}
$$

where $\psi=2 \gamma_{p l} ; \theta_{p, c 1}=\operatorname{Min}\left(\phi_{a p}, 0.5 \pi-\psi\right)$ is a critical angle, for radiation incident at $\theta_{p}>\theta_{p, c 1}$, radiation irradiating on right plane wall will not arrive on solar cells. The $\phi_{a p}$ (see Figure $5 \mathrm{~d}$ ) is given by:

$$
\tan \phi_{a p}=a\left(0.5 C_{t}+0.5+\cos \psi\right) /(h+a \sin \psi)
$$

As shown in Figure 5a, incident angle of solar rays on solar cells can be simply calculated based on vector $n_{r}$ and vector of the normal to image $B A^{\prime}$ as:

$$
\cos \theta_{i n, 2}=n_{r} \cdot(\cos \psi, 0, \sin \psi)=n_{x 1} \cos \psi+n_{z 1} \sin \psi
$$

As seen from Figure 5, the path length of solar ray from the aperture to solar cells is different for different solar rays, thus $\tau_{a}=\exp (-k L)$ is different. However, the $k L$ is small for DCPVs with small size of solar cells, and in this case $\tau_{a} \approx 1-k L$, implying that $\tau_{a}$ is approximately a linear function of path length $L$, therefore, $\tau_{a, 2}=\exp \left(-k L_{2}\right)$ in Equation (21) can be estimated based on average path length of all solar rays as follows.

For $\theta_{p} \leq \theta_{a}$ 
As shown in Figure 5a, radiation incident on the entire plane wall is reflected onto solar cells in this case. Hence the average path length $L_{2}$ is given by:

$$
L_{2}=\left(l_{1}+l_{3}+l_{2}\right) / 2=\left(h / \cos \theta_{r}-0.5 x_{D} / \cos \theta_{r}+0.5 x_{D} / \cos \theta_{i n, 2}\right)
$$

For $\theta_{a}<\theta_{p} \leq \theta_{t}$

In this case, the radiation incident on the lower part (BM) of plane wall is reflected onto solar cells as shown in Figure $5 b$. The position of $\mathrm{M}$ is subject to the following equation:

$$
\left\{\begin{array}{l}
(0.5 a+a \cos \psi-z) /(x+a \sin \psi)=\tan \theta_{p} \\
(z-0.5 a) / x=\tan \gamma_{p l}
\end{array}\right.
$$

which leads to:

$$
x_{M}=a\left(\cos \psi-\tan \theta_{p} \sin \psi\right) /\left(\tan \theta_{p}+\sin \gamma_{p l}\right)
$$

Therefore, one has $l_{1}=\left(h-x_{M}\right) / \cos \theta_{r} ; l_{2}=h / \cos \theta_{r} ; l_{3}=x_{M} / \cos \theta_{i n, 2}$, thus:

$$
L_{2}=\left(l_{1}+l_{2}+l_{3}\right) / 2=\left(2 h / \cos \theta_{r}-x_{M} / \cos \theta_{r}+x_{M} / \cos \theta_{i n, 2}\right) / 2
$$

For $\theta_{t}<\theta_{p}<\theta_{p, c 1}$

As seen from Figure $5 c$, radiation incident on middle part (MN) of the plane wall is reflected onto solar cells. Similar to find $x_{M}$, one has:

$$
x_{N}=\left(h \tan \theta_{p}-0.5 a-0.5 a C_{t}\left(\tan \theta_{p}+\sin \gamma_{p l}\right)\right.
$$

Thus one has:

$$
L_{2}=\left(l_{1}+l_{2}+l_{3}+l_{4}\right) / 2=\left(2 h / \cos \theta_{r}-x_{M} / \cos \theta_{r}+x_{M} / \cos \theta_{i n, 2}+x_{N} / \cos \theta_{i n, 2}\right) / 2
$$

As shown in Figure 6, solar rays irradiating the plane wall or the lower parabolic wall are leaked as $\theta_{i, p l}<\theta_{c}$, and the energy fraction of radiation loss due to partial internal reflection is given by:

$$
f_{L, 1}=\tau \tau_{a, 2} \Delta z_{2}\left(1-\rho\left(\theta_{i, p l}\right)\right) / a C_{t}
$$

The photovoltaic efficiency loss of DCPVs due to radiation leakage is calculated by:

$$
\eta_{L, 1}=f_{L, 1} \eta_{p v}\left(\theta_{i n, 2}\right)
$$

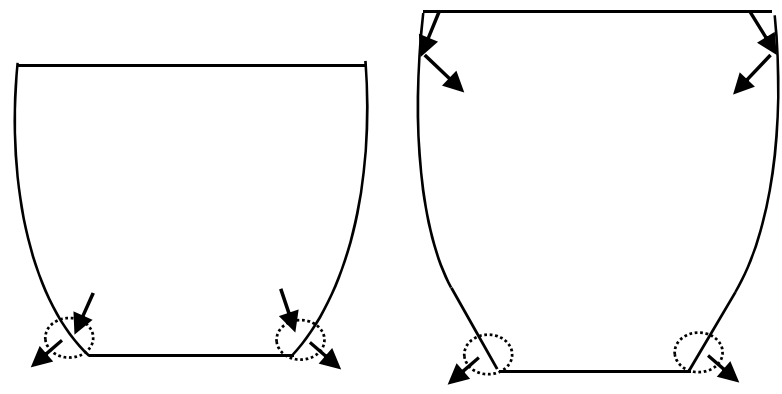

Figure 6. (Left) leakage of radiation irradiating on lower walls of DCPV- $\theta_{a} / \theta_{e}$ for $\theta_{p} \leq \theta_{a}$; (Right) leakage of radiation irradiating on upper parabolic walls for $\theta_{p}>\theta_{a}$. 


\subsubsection{Calculation of $f_{3}$ and $\eta_{3}$}

As seen from Figure 7, the left plane wall is fully and partially irradiated for $\theta_{p} \leq \gamma_{f d}$ and $\gamma_{f d}<\theta_{p}<\gamma_{f}$, respectively.

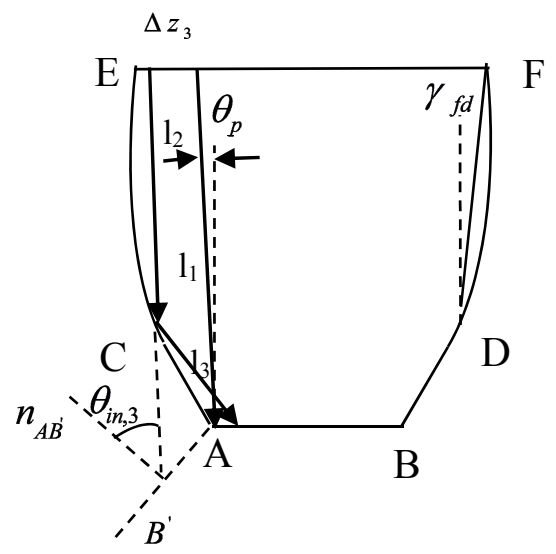

Figure 7. Irradiation situation of left plane wall as $\theta_{p} \leq \gamma_{f d}$.

The energy fraction of radiation irradiating on left plane wall and arriving on solar cells is as follows ( $\rho=1$ in this case as aforementioned):

$$
\begin{gathered}
f_{3}=\Delta z_{3} \tau \tau_{a, 3} / a C_{t} \\
\Delta z_{3}= \begin{cases}z_{D}-0.5 a-x_{D} \tan \theta_{p} & \theta_{p} \leq \gamma_{f d} \\
0.5\left(C_{t}-1\right) a-h \tan \theta_{p} & \gamma_{f d}<\theta_{p}<\gamma_{f} \\
0 & \theta_{p} \geq \gamma_{f}\end{cases} \\
\tan \gamma_{f d}=\left(0.5 a C_{t}-z_{D}\right) /\left(h-x_{D}\right)
\end{gathered}
$$

As shown in Figure 7 , the solar incident angle, $\theta_{i n, 3}$, is given by:

$$
\cos \theta_{i n, 3}=n_{r} \cdot n_{A B^{\prime}}=n_{x 1} \cos \psi-n_{z 1} \sin \psi
$$

where $n_{A B^{\prime}}=(\cos \psi, 0,-\sin \psi)$ is the vector of normal to image $A B^{\prime}$. The average path length of solar rays from the aperture to solar cells for calculating $\tau_{a, 3}=\exp \left(-k L_{3}\right)$ is given by:

$$
\begin{gathered}
L_{3}= \begin{cases}\left(h-0.5 x_{D}\right) / \cos \theta_{r}+0.5 x_{D} / \cos \theta_{i n, 3} & \left(\theta_{p} \leq \gamma_{f d}\right) \\
\left(h-0.5 x_{M}\right) / \cos \theta_{r}+0.5 x_{M} / \cos \theta_{i n, 3} & \left(\gamma_{f d}<\theta_{p}<\gamma_{f}\right)\end{cases} \\
x_{M}=\left[0.5 a\left(C_{t}-1\right)-h \tan \theta_{p}\right] /\left(\tan \gamma_{p l}-\tan \theta_{p}\right)
\end{gathered}
$$

Similarly, one has:

$$
\eta_{3}=f_{3} \eta_{p v}\left(\theta_{i n, 3}\right)
$$

\subsubsection{Method for calculating $f_{4}$ and $\eta_{4}$}

To find the radiation irradiating on the right parabolic wall at $\theta_{p} \leq \theta_{a}$ and arriving on solar cells $\left(I_{4}\right)$, the finite element method is employed. As shown in Figure 8, solar rays incident on a finite element around $M$ at $\theta_{p} \leq \theta_{a}$ will arrive on solar cells after more than one reflection. The reflectivity of solar ray at $\mathrm{M}$ is dependent on its incident angle $\theta_{i, M}$, but the reflectivity of solar ray at point $N$ for next reflection is 1 [31]. Hence, radiation on solar cells from finite element around $M$ is given by:

$$
\Delta I_{4}=\tau \tau_{a, 4} \delta z_{4} \rho\left(\theta_{i, M}\right)
$$


The $\delta z_{4}$ in the above expression is given by:

$$
\delta z_{4}=-\left(d z+d x \tan \theta_{p}\right)
$$

The power output due to contribution of $\Delta I_{4}$ is as:

$$
\Delta P_{4}=\Delta I_{4} \eta_{p v}\left(\theta_{i n, 4}\right) \tau \tau_{a, 4} \delta z_{4} \rho\left(\theta_{i, M}\right) \eta_{p v}\left(\theta_{i n, 4}\right)
$$

As shown in Figure 8, path length $L_{4}$ of solar ray depends on vector $r_{M}$, and $r_{M}$ is given by [13,41]:

$$
r_{M}=-n_{r}+2\left(n_{r} \cdot n_{M}\right) n_{M}=\left(-n_{x 1} \cos 2 \gamma_{M}-n_{z 1} \sin 2 \gamma_{M},-n_{y 1},-n_{x 1} \sin 2 \gamma_{M}+2 n_{z 1} \cos 2 \gamma_{M}\right)
$$

The point $N$, where $r_{M}$ hits, is determined by projected angle $\left(\theta_{p, M}\right)$ of $r_{M}$ and $\varphi_{M}$ of point $M$. The $\theta_{p, M}$ is given by:

$$
\theta_{p, M}=\theta_{p}+2 \gamma_{M}
$$

The position of point $M$ relative to $D$ and $B$ (see Figure 8 ) can be represented by the tilt-angles of lines $M D$ and $M B$ as:

$$
\begin{gathered}
\tan \gamma_{M D}=\left(z_{M}-z_{D}\right) /\left(x_{M}-x_{D}\right) \\
\tan \gamma_{M B}=\left(z_{M}-0.5 a\right) / x_{M}
\end{gathered}
$$

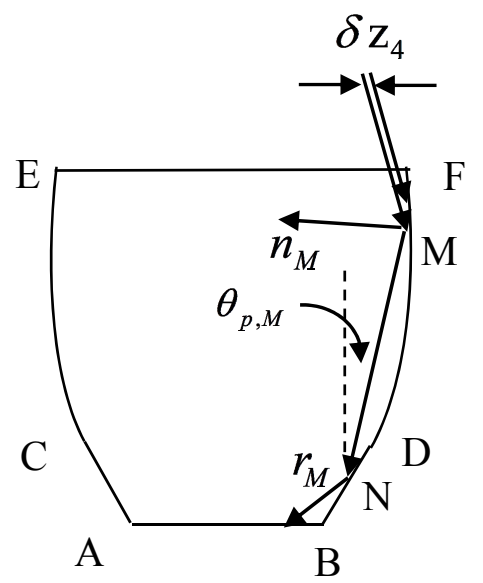

Figure 8. The way to solar cells for radiation incident on point $\mathrm{M}$ of right parabola at $\theta_{p} \leq \theta_{a}$.

For $\theta_{p, M} \geq \gamma_{M B}$

As shown in Figure 8, $r_{M}$ directly hits on solar cells when $\theta_{p, M} \geq \gamma_{M B}$, and path length $L_{4}$ in this case is given by:

$$
L_{4}=\left(h-x_{M}\right) / \cos \theta_{r}+x_{M} / \cos \theta_{r, M}
$$

and $\theta_{i n, 4}=\theta_{r, M}$. The exit angle of $r_{M}$ is given by:

$$
\cos \theta_{r, M}=-r_{M} \cdot(1,0,0)=n_{x 1} \cos 2 \gamma_{M}+n_{z 1} \sin 2 \gamma_{M}
$$

For $\gamma_{M D} \leq \theta_{p, M}<\gamma_{M B}$

In this case, $r_{M}$ hits on the plane wall (see Figure 8) first and then redirects onto solar cells. The vector $\left(r_{N}\right)$ of solar ray reflecting from $\mathrm{N}$ and solar incident angle $\left(\theta_{i n, 4}\right)$ are subject to:

$$
\left\{\begin{array}{l}
r_{N}=-i_{N}+2\left(i_{N} \cdot n_{p l}\right) n_{p l} \\
i_{N}=-r_{M} \\
\cos \theta_{i n, 4}=-r_{N} \cdot(1,0,0)
\end{array}\right.
$$


where $n_{p l}=\left(\sin \gamma_{p l}, 0,-\cos \gamma_{p l}\right)$ is the unit vector of normal to right plane wall, thus one obtains:

$$
\cos \theta_{i n, 4}=n_{x 1} \cos 2\left(\gamma_{p l}-\gamma_{M}\right)-n_{z 1} \sin 2\left(\gamma_{p l}-\gamma_{M}\right)
$$

The position of point $N$ is subject to:

$$
\left\{\begin{array}{l}
\tan \theta_{p, M}=\left(z_{M}-z_{N}\right) /\left(x_{M}-x_{N}\right) \\
\tan \gamma_{p l}=\left(z_{N}-0.5 a\right) / x_{N}
\end{array}\right.
$$

Thus one obtains:

$$
x_{N}=\left(z_{M}-0.5 a-x_{M} \tan \theta_{p, M}\right) /\left(\tan \gamma_{p l}-\tan \theta_{p, M}\right)
$$

The path length of solar ray in this case is calculated by:

$$
L_{4}=\left(h-x_{M}\right) / \cos \theta_{r}+\left(x_{M}-x_{N}\right) / \cos \theta_{r, M}+x_{N} / \cos \theta_{i n, 4}
$$

For $\theta_{p, M}<\gamma_{M D}$

In this case, $r_{M}$ hits on lower parabolic wall $(M D)$. The $\varphi_{N}$ of point $N$ is determined by:

$$
\tan \theta_{p, M}=\left(x_{M}-x_{N}\right) /\left(z_{M}-z_{N}\right)
$$

Given $\varphi_{M}, x_{M}$ and $z_{M}$ are calculated by Equation (1), then $\varphi_{N}$ is obtained by substituting Equation (1) into Equation (53) for $z_{N}$ and $x_{N}$, and the tilt-angle $\left(\gamma_{N}\right)$ of the line tangent to the parabola at point $N$ can be obtained based on Equation (10) by setting $\varphi_{M}=\varphi_{N}$. Previous studies showed that the fraction of radiation that arrives on the solar cells of CPV after more than three reflections is considerably small, and the two-reflection model can accurately predict the optical performance of CPV [10,13], thus any further reflections of solar rays reflecting from $\mathrm{N}$ is not considered in this work. Hence, the exit angle of $r_{N}$ is regarded as the incident angle of solar rays on solar cells and can be calculated based on Equation (49) by replacing $\gamma_{p l}$ with $r_{N}$, and the path length of solar ray $\left(L_{4}\right)$ can be estimated based on Equation (52) and $\varphi_{N}$ obtained here.

The total radiation that incident on right parabolic wall and arriving on solar cells of DCPVs, $I_{4}$, can be calculated by integrating $\Delta I_{4}$ from $\varphi=\theta_{t}$ to $\theta_{e}$, thus $f_{4}$ and $\eta_{4}$ are calculated as follows:

$$
\begin{gathered}
f_{4}=-\int_{\varphi=\theta_{t}}^{\varphi=\theta_{e}}\left(d z+d x \tan \theta_{p}\right) \tau \tau_{a, 4} \rho\left(\theta_{i, M}\right) / a C_{t} \\
\eta_{4}=-\int_{\phi=\theta_{t}}^{\phi=\theta_{e}}\left(d z+d x \tan \theta_{p}\right) \tau \tau_{a, 4} \rho\left(\theta_{i, M}\right) \eta_{p v}\left(\theta_{i n, 4}\right) / a C_{t}
\end{gathered}
$$

The energy fraction of radiation loss due to leakage from parabolic wall is given by:

$$
f_{L, 2}=-\int_{\phi=\theta_{t}}^{\phi=\theta_{e}}\left(d z+d x \tan \theta_{p}\right) \tau \tau_{a, 4}\left(1-\rho\left(\theta_{i, M}\right)\right) / a C_{t}
$$

The photovoltaic efficiency loss of DCPVs due to radiation leakage is calculated by:

$$
\eta_{L, 2}=-\int_{\varphi=\theta_{t}}^{\varphi=\theta_{e}}\left(d z+d x \tan \theta_{p}\right) \tau \tau_{a, 4}\left(1-\rho\left(\theta_{i, M}\right)\right) \eta_{p v}\left(\theta_{i n, 4}\right) / a C_{t}
$$

It is noted that $f_{4}, f_{L, 2}, \eta_{4}$ and $\eta_{L, 2}$ are zero for $\theta_{p}>\theta_{a}$ because no radiation reflecting from right parabolic wall arrives on solar cells when $\theta_{p}>\theta_{a}$. 


\subsubsection{Calculation of $f_{5}$ and $\eta_{5}$}

As shown in Figure 9, the left parabolic wall is fully irradiated for $\theta_{p} \leq \gamma_{a p}$, and partially irradiated as $\gamma_{a p}<\theta_{p}<\gamma_{f d}\left(\gamma_{a p}\right.$ is the tilt-angle of the line tangent to parabolic wall at upper ends and calculated based Equation (10) by setting $\varphi_{M}=\theta_{t}$ ). For $\gamma_{a p}<\theta_{p, M}<\gamma_{f d}$, the lower left parabolic wall (VC) is irradiated, and $\varphi_{V}$ of point $\mathrm{V}$ is determined by:

$$
\tan \theta_{p}=\left(0.5 C_{t}-z_{V}\right) /\left(h-x_{V}\right)
$$

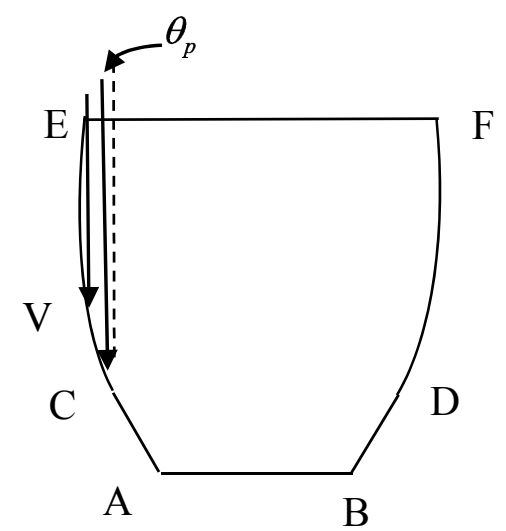

Figure 9. Irradiation situation of left parabolic wall when $\gamma_{a p}<\theta_{p}<\gamma_{f d}$.

It is noted that $\varphi_{V}=\theta_{t}$ for $\theta_{p} \leq \gamma_{a p}$. For symmetric DCPVs, the optical and photovoltaic performance are identical for radiation incident at $\pm \theta_{p}$. Therefore, $f_{5}$ and $\eta_{5}$ can be calculated based on the method to find $f_{4}$ and $\eta_{4}$ by setting $\theta_{p}=-\theta_{p}, n_{z 1}=\left|n_{z 1}\right|$ and $\rho\left(\theta_{i, M}\right)=1$ as follows:

$$
\begin{gathered}
f_{5}=-\int_{\varphi=\varphi_{V}}^{\varphi=\theta_{e}}\left(d z-d x \tan \theta_{p}\right) \tau \tau_{a, 5} / a C_{t} \\
\eta_{5}=-\int_{\varphi=\varphi_{V}}^{\varphi=\theta_{e}}\left(d z-d x \tan \theta_{p}\right) \tau \tau_{a, 5} \eta_{p v}\left(\theta_{i n, 5}\right) / a C_{t}
\end{gathered}
$$

It is noted that no solar leakage takes place for this case, and $f_{5}, \eta_{5}$ are zero for $\theta_{p} \geq \gamma_{f d}$ because the left parabolic wall is completely shaded by itself for $\theta_{p} \geq \gamma_{f d}$.

\subsubsection{Calculation of $f_{6}$ and $\eta_{6}$}

As shown in Figure 10, solar rays striking at upper end $(F)$ of right parabolic wall at $\theta_{p}=\theta_{p, c 2}$ just redirect to the end $\left(B^{\prime}\right)$ of solar cells' image first then redirect to end $(B)$ of solar cells. Thus, refractive rays irradiating on the upper right parabola $(F K)$ at $\theta_{a}<\theta_{p}<\theta_{p, c 2}$ will arrive on solar cells after two reflections. The critical angle $\theta_{p, c 2}$ is subject to:

$$
\theta_{p, c 2}=\phi_{a p}-2 \gamma_{a p}
$$

The $\varphi_{K}$ of critical point $K$ is subject to:

$$
\left\{\begin{array}{l}
\theta_{p, K}=2 \gamma_{K}+\theta_{p} \\
\tan \gamma_{K}=\left(\cos \theta_{a}-\cos \phi_{K}\right) /\left(\sin \theta_{a}+\sin \phi_{K}\right) \\
\tan \theta_{p, K}=\left(z_{K}+0.5 a+a \cos \psi\right)\left(x_{K}+a \sin \psi\right)
\end{array}\right.
$$




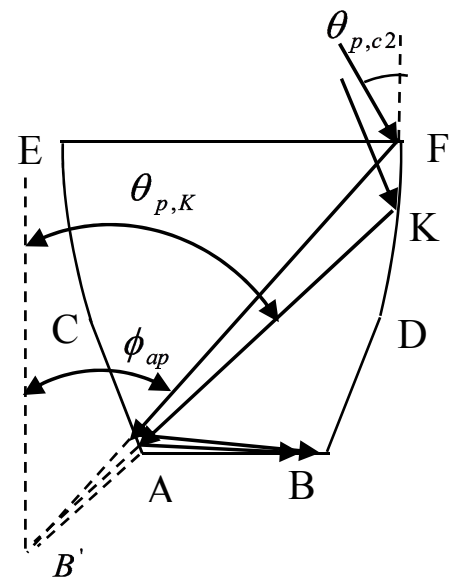

Figure 10. Critical angle $\theta_{p, c 2}$ and critical point $K$ for $\theta_{p}>\theta_{a}$.

As shown in Figure 11, coordinates of point $N$, where $r_{M}$ hits, are subject to:

$$
\left\{\begin{array}{l}
\tan \theta_{p, M}=\left(z_{M}-z_{N}\right) /\left(x_{M}-x_{N}\right) \\
\tan \gamma_{p l}=\left(-0.5 a-z_{N}\right) / x_{N}
\end{array}\right.
$$

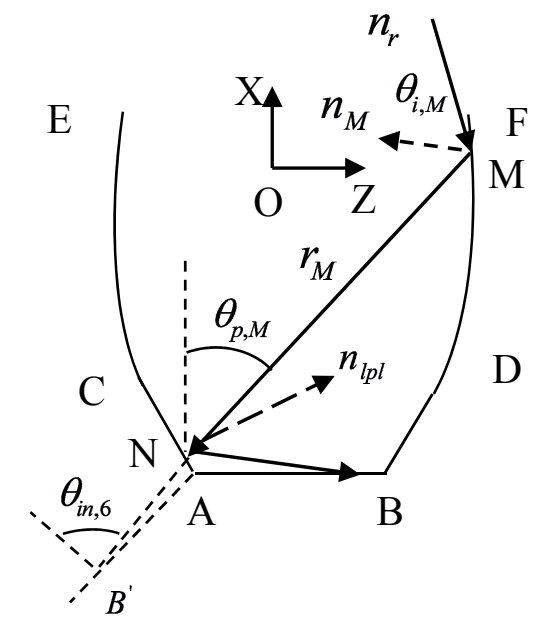

Figure 11. Way to solar cells for radiation incident on upper parabola wall at $\theta_{a}<\theta_{p}<\theta_{p, c 2}$.

Thus, one has:

$$
x_{N}=\left(x_{M} \tan \theta_{p, M}-z_{M}-0.5 a\right) /\left(\tan \gamma_{p l}+\tan \theta_{p, M}\right)
$$

in which $\theta_{p, M}=\theta_{p}+2 \gamma_{M}$. The vector of normal to left plane wall is expressed by:

$$
n_{l p l}=\left(\sin \gamma_{p l}, 0, \cos \gamma_{p l}\right)
$$

The incidence angle of a solar ray on the left plane wall is as:

$$
\cos \theta_{i, N}=-r_{M} \cdot n_{l p l}=\cos \theta_{r, M} \sin \gamma_{p l}+\left(n_{x 1} \sin 2 \gamma_{M}-n_{z 1} \cos 2 \gamma_{M}\right) \cos \gamma_{p l}
$$

As shown in Figure 11, the incidence angle on solar cells, $\theta_{i n, 6}$, is given by:

$$
\cos \theta_{i n, 6}=-r_{M} \cdot n_{A B^{\prime}}=n_{x 1} \cos \left(\psi+2 \gamma_{M}\right)+n_{z 1} \sin \left(\psi+2 \gamma_{M}\right)
$$


The path length $L_{6}$ for calculating $\tau_{a, 6}=\exp \left(-k L_{6}\right)$ is given by:

$$
L_{6}=\left(h-x_{M}\right) / \cos \theta_{r}+\left(x_{M}-x_{N}\right) / \cos \theta_{r, M}+x_{N} / \cos \theta_{i n, 6}
$$

The energy of radiation incident on a finite element around $M$ and arriving on solar cells after two reflections is calculated by:

$$
\Delta I_{6}=-\left(d z+d x \tan \theta_{p}\right) \tau \tau_{a, 6} \rho\left(\theta_{i, M}\right) \rho\left(\theta_{i, N}\right)
$$

The electricity generated by $\Delta I_{6}$ is given by:

$$
\Delta P_{6}=\Delta I_{6} \eta_{p v}\left(\theta_{i n, 6}\right)=-\left(d z+d x \tan \theta_{p}\right) \tau \tau_{a, 6} \rho\left(\theta_{i, M}\right) \rho\left(\theta_{i, N}\right) \eta_{p v}\left(\theta_{i n, 6}\right)
$$

where $\rho\left(\theta_{i, M}\right)$ and $\rho\left(\theta_{i, N}\right)$ are the reflectivity of solar rays at $M$ and $N$, respectively, and determined by their incidence angles. The total radiation, $I_{6}$, can be estimated by integrating Equation (68) from $\varphi=$ $\theta_{t}$ to $\varphi_{K}$, thus $f_{6}$ and $\eta_{6}$ are calculated by:

$$
\begin{gathered}
f_{6}= \begin{cases}-\int_{\theta_{t}}^{\varphi_{K}}\left(d z+d x \tan \theta_{p}\right) \tau \tau_{a, 6} \rho\left(\theta_{i, M}\right) \rho\left(\theta_{i, N}\right) / a C_{t} & \left(\theta_{a}<\theta_{p}<\theta_{p, c 2}\right) \\
0 & \text { else }\end{cases} \\
\eta_{6}= \begin{cases}-\int_{\theta_{t}}^{\varphi_{K}}\left(d z+d x \tan \theta_{p}\right) \tau \tau_{a, 6} \rho\left(\theta_{i, M}\right) \rho\left(\theta_{i, N}\right) \eta_{p v}\left(\theta_{i n, 6}\right) / a C_{t} & \left(\theta_{a}<\theta_{p}<\theta_{p, c 2}\right) \\
0 & \text { else }\end{cases}
\end{gathered}
$$

The energy fraction of radiation loss due to partial internal reflections (see Figure 6) is given by:

$$
f_{L, 3}= \begin{cases}-\int_{\theta_{t}}^{\varphi_{K}}\left(d z+d x \tan \theta_{p}\right) \tau \tau_{a, 6}\left(1-\rho\left(\theta_{i, M}\right) \rho\left(\theta_{i, N}\right)\right) / a C_{t} & \left(\theta_{a}<\theta_{p}<\theta_{p, c 2}\right) \\ 0 & \text { else }\end{cases}
$$

The photovoltaic efficiency loss due to solar leakage is calculated by:

$$
\eta_{L, 3}= \begin{cases}-\int_{\theta_{t}}^{\varphi_{K}}\left(d z+d x \tan \theta_{p}\right) \tau \tau_{a, 6}\left(1-\rho\left(\theta_{i, M}\right) \rho\left(\theta_{i, N}\right)\right) \eta_{p v}\left(\theta_{i n, 6}\right) / a C_{t} & \left(\theta_{a}<\theta_{p}<\theta_{p, c 2}\right) \\ 0 & \text { else }\end{cases}
$$

The analysis in the above shows that, given the geometry of DCPV- $\theta_{a} / \theta_{e}, n_{r}$ at any time of a day can be determined, then $f, f_{L}=f_{L, 1}+f_{L, 2}+f_{L, 3}, \eta$ and $\eta_{L}=\eta_{L, 1}+\eta_{L, 2}+\eta_{L, 3}$ are obtained.

\section{Annual Optical and Photovoltaic Performance of DCPVs}

It is assumed that the length of DCPV- $\theta_{a} / \theta_{e}$ is infinite as compared to the width, and radiation from the ground is not considered, thus, radiation received by unit area of solar cells at any time of a day is calculated by:

$$
I=C_{t} I_{b} g\left(\theta_{a p}\right) n_{x} f+I_{a b s, d}
$$

The electricity from a unit area of solar cells of DCPVs at any time is expressed by:

$$
P=C_{t} I_{b} g\left(\theta_{a p}\right) n_{x} \eta+P_{d}
$$

where $I_{b}$ is the intensity of beam radiation; $g\left(\theta_{a p}\right)$ is a control function, being 1 for $\cos \theta_{a p}>0$ otherwise zero. The $I_{a b s, d}$ in Equation (73) is the sky diffuse radiation received by unit area of solar cells, and $P_{d}$ in Equation (74) is the electricity generated by $I_{a b s, d}$. For isotropic sky diffuse radiation, they are calculated by [13]:

$$
I_{a b s, d}=\frac{2 C_{t} I_{d}}{\pi} \int_{\phi_{0}}^{\pi} d \phi \int_{0}^{0.5 \pi} \sin \theta \cos \theta f d \theta=C_{d} I_{d}
$$




$$
P_{d}=\frac{2 C_{t} I_{d}}{\pi} \int_{\phi_{0}}^{\pi} d \phi \int_{0}^{0.5 \pi} \sin \theta \cos \theta \eta d \theta=C_{d, p} I_{d}
$$

where:

$$
\begin{aligned}
C_{d} & =\frac{2 C_{t}}{\pi} \int_{\phi_{0}}^{\pi} d \phi \int_{0}^{0.5 \pi} \sin \theta \cos \theta f d \theta \\
C_{d, p} & =\frac{2 C_{t}}{\pi} \int_{\phi_{0}}^{\pi} d \phi \int_{0}^{0.5 \pi} \sin \theta \cos \theta \eta d \theta
\end{aligned}
$$

The $\phi_{0}$ is related to $\theta$ by [13]:

$$
\begin{cases}\phi_{0}=0 & (\theta \leq 0.5 \pi-\beta) \\ \tan \phi_{0}=1 /(\tan \theta \tan \beta) & (0.5 \pi-\beta<\theta \leq 0.5 \pi)\end{cases}
$$

Given $\beta$ and geometry of DCPVs, $C_{d}$ and $C_{d, p}$ are constants. Hence, the daily radiation received by unit area of solar cells of DCPVs is calculated by integrating Equation (73) over the daytime as:

$$
H_{\text {day }}=C_{t} \int_{-t_{0}}^{t_{0}} I_{b} g\left(\theta_{a p}\right) n_{x} f d t+C_{d} H_{d}
$$

The daily electricity from DCPVs is given by integrating Equation (74) over the daytime:

$$
P_{\text {day }}=C_{t} \int_{-t_{0}}^{t_{0}} I_{b} g\left(\theta_{a p}\right) n_{x} \eta d t+C_{d, p} H_{d}
$$

The daily radiation loss $\left(H_{\text {day }, L}\right)$ and power loss $\left(P_{\text {day }, L}\right)$ due to solar leakage can be calculated based on Equations (80) and (81) by replacing $f$ and $\eta$ with $f_{L}$ and $\eta_{L}$, respectively. Daily radiation on unit area of aperture or similar PV panels is expressed by:

$$
H_{\text {day }, 0}=\int_{-t_{0}}^{t_{0}} I_{b} g\left(\theta_{a p}\right) n_{x} d t+C_{d 0, p} H_{d}
$$

Daily electricity from similar non-concentrating PV panels is given by:

$$
P_{\text {day }, 0}=\int_{-t_{0}}^{t_{0}} I_{b} g\left(\theta_{a p}\right) n_{x} \eta_{p v}\left(\theta_{a p}\right) d t+C_{d 0, p} H_{d}
$$

$C_{d 0}$ and $C_{d 0, p}$ can be calculated based on Equations (77) and (78) by setting $f=1$ and $\eta=\eta_{p v}\left(\theta_{a p}\right)$, respectively. The $H_{d}$ in above expressions is the daily sky diffuse radiation on the horizon, and $\mathrm{t}_{0}$ is the sunset time on the horizon.

At any time of a day, the position of the sun in terms of $n_{s}$ can be determined, then $f, f_{L}, \eta$ and $\eta_{L}$ can be calculated. Therefore, given $H_{d}$ and time variation of $I_{b}$ in a day, $H_{\text {day }, 0}, H_{\text {day }}, H_{\text {day }, L}, P_{\text {day }, 0}, P_{\text {day }}$ and $P_{\text {day, } L}$ can be numerically obtained, then summing them for all days of a year gives the annual radiation on the aperture $\left(S_{0}\right)$, annual radiation collected by unit area of solar cells of DCPVs $\left(S_{a}\right)$, annual radiation loss due to leakage $\left(S_{L}\right)$, annual power output from similar PV panel $\left(P_{o}\right)$, annual electricity from $\operatorname{DCPV}\left(P_{a}\right)$, and annual power loss due to leakage $\left(P_{L}\right) . S_{a}\left(\theta_{x}\right)$, the annual radiation on solar cells at incidence angles larger than $\theta_{x}$, can be simply calculated by setting $I_{i}$ or $\Delta I_{i}=0$ in relevant expressions when $\theta_{i n, i}<\theta_{x}$. Compared to similar PV panel, the annual average solar gain and power increase factors of DCPVs are given by:

$$
\begin{gathered}
C_{s}=\frac{S_{a}}{S_{0}}=\frac{S_{a}}{S_{0} C_{t}} C_{t}=f_{a} C_{t} \\
C_{p}=\frac{P_{a}}{P_{0}}=\frac{P_{a}}{S_{a}} \times \frac{S_{a}}{S_{0} C_{t}} \times \frac{S_{0} C_{t}}{P_{0}}=f_{a} \eta_{a} / \eta_{a, 0}=f_{a} C_{p v} C_{t}
\end{gathered}
$$


where $f_{a}=S_{a} / S_{0} C_{t}$ is the annual average optical efficiency of DCPVs, $\eta_{a}=P_{a} / S_{a}$ and $\eta_{a, 0}=P_{0} / S_{0}$ are the annual average photovoltaic efficiency of solar cells for concentrating and non-concentrating radiation, respectively. Hence $C_{p v}=\eta_{a} / \eta_{a, 0}$ represents the electricity loss coefficient of DCPVs due to increased the incidence angle of solar rays on solar cells after reflections from walls. It is known from Equation (85) that $f_{a} C_{p v}$ is an indication to represent the overall performance of DCPVs, for a perfect $\mathrm{DCPV}$, the power increase factor $C_{p}$ is equal to $C_{t}$, thus $f_{a} C_{p v}=1$, but in practice, $C_{p}$ is much less than $C_{t}$, hence $f_{a} C_{p v}$ much less than 1 due to optical loss and electricity loss. For DCPVs, there is no optical loss due to reflections, and optical loss is attributed to solar absorption and leakage.

In this work, the monthly horizontal radiation averaged over many years in Beijing $\left(\lambda=39.95^{\circ}\right)$ was used for calculations [49], and monthly average daily sky diffuse radiation on the horizon $\left(H_{d}\right)$ and the time variation of $I_{b}$ in a day are estimated based on correlations proposed by Collares-Pereira and Rabl [50]. DCPVs with $n=1.5, k=4 \mathrm{~m}^{-1}$ and $a=3 \mathrm{~mm}$ are investigated except with a specific indication. The sunset time on the horizon in a day is calculated based on declination of the Sun in the day. The step of $\varphi$ for calculating $f$ and $\eta$ is set to be $0.1^{\circ}$, and step of $\theta$ and $\phi$ for calculating $C_{d}$ and $C_{d, p}$ is taken to be $0.1^{\circ}$; the time interval to calculate daily radiation collection and daily electricity generation is set to be $1 \mathrm{~min}$. The visual basic code programmed based on mathematical expressions presented here is used for calculations. To fully investigate effects of geometry of DCPV- $\theta_{a} / \theta_{e}$ on the performance, DCPVs with the aperture's tilt-angle being yearly fixed (1T-DCPVs), yearly adjusted two times at two-tilts (2T-DCPVs) and yearly adjusted four times at three tilts (3T-DCPVs) are considered. For 1T-DCPVs, $\beta=\lambda$; for 2T-DCPVs, $\beta$ is set to be $\lambda-18^{\circ}$ and $\lambda+18^{\circ}$ in summers and winters, respectively; whereas for 3T-DCPVs, $\beta$ is set to be $\lambda$ during the periods of 23 days around both equinoxes, and adjusted to be $\lambda-22^{\circ}$ and $\lambda+22^{\circ}$ in summers and winters, respectively [31].

\section{Results and Discussions}

\subsection{Radiation Leakage from Walls}

Figure 12 presents the energy fraction of radiation lost due to leakage from the walls of 1T-DCPV-18/90 and 1T-DCPV-18/85 in solstices. It is seen that, for 1T-DCPV-18/90, a fraction of radiation incident on the lower parabolic wall leaks during the time around solar-noon because the minimum solar incident angle on the wall occurs at solar-noon [31]; whereas for 1T-DCPV-18/65, leakage takes place from 15:40 to 16:30 because $\theta_{a}<\theta_{p}<\theta_{p, c 2}$ and radiation irradiating on the upper parabolic wall redirects to the opposite plane wall first then leaks to air as shown in right of Figure 6. This means that the use of DCPC $-\theta_{a} / \theta_{e}$ with $\theta_{e}<90$ can reduce leakage of radiation within its acceptance angle, but it results in a leakage for radiation outside its acceptance angle.
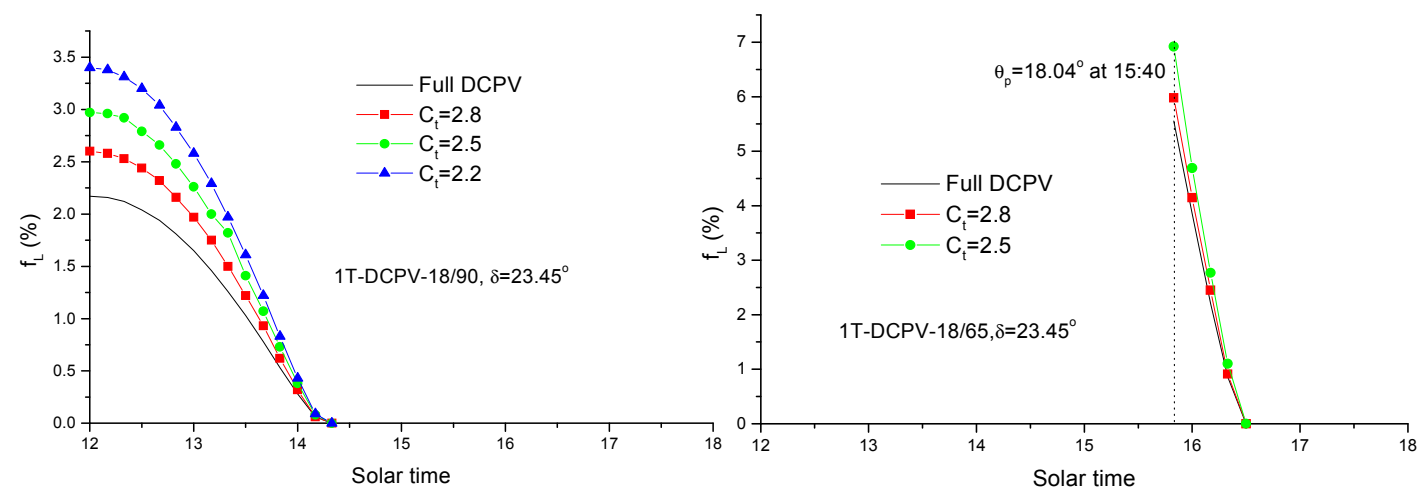

Figure 12. Solar leakage loss of 1T-DCPV-18/90 (left) and 1T-DCPV-18/65 (right) in solstices.

Figure 13 shows effects of $\theta_{e}$ on annual leakage loss of 1T-DCPV-18/ $\theta_{e}$. It is seen that, with the increase of $\theta_{e}, S_{L, 3}$ decreases but $S_{\mathrm{L}, 2}$ increases, and this is a result of the fact that, with the increase of $\theta_{e}$, less radiation irradiating on upper parabolic walls at $\theta_{a}<\theta_{p}<\theta_{p, c 2}$ leaks through the opposite 
plane wall, meanwhile more radiation irradiating on lower parabolic wall at $\theta_{p}<\theta_{a}$ is leaked. It is also seen from Figure 13 that, for full DCPVs (left), $\mathrm{S}_{\mathrm{L}, 1}$, annual leakage loss of radiation irradiating on plane walls, is zero for $\theta_{e}<77^{\circ}$ and increases with $\theta_{e}$ for $\theta_{e}<87^{\circ}$ then decreases; whereas for truncated DCPVs (right), it decreases with $\theta_{e}$ for $\theta_{e}<83.6^{\circ}$ then increases for $83.6^{\circ}<\theta_{e}<87^{\circ}$ and finally decrease. This is because for full DCPVs, all beam and sky diffuse radiation irradiating on the plane walls is totally internally reflected as $\theta_{e}<77^{\circ}$ and all leakage loss is attributed to the radiation irradiating on upper parabolic walls at $\theta_{a}<\theta_{p}<\theta_{p, c 2}$ as shown in Figure 14; whereas for truncated DCPVs, a fraction of sky diffuse radiation irradiating on plane walls is not totally internally reflected as $\theta_{e}<77^{\circ}$ although direct sunlight irradiating on plane walls can be totally internally reflected for $\theta_{e}<83.6^{\circ}$ [31].
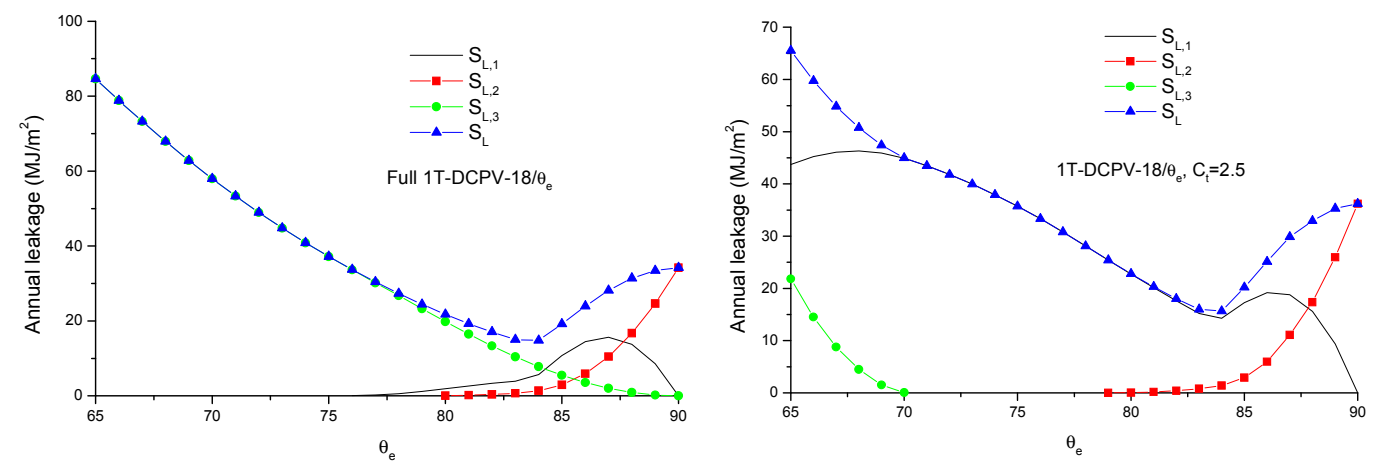

Figure 13. Variations of annual leakage loss with $\theta_{e}$ for 1T-DCPV-18/ $\theta_{e}$.

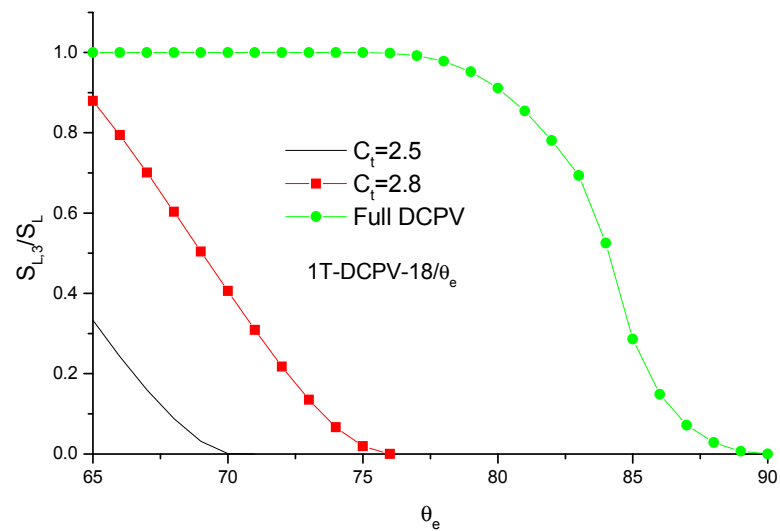

Figure 14. Effects of $\theta_{e}$ on $S_{L, 3}$, leakage loss for radiation irradiating on the upper parabolic wall at $\theta_{a}<\theta_{p}<\theta_{p, c 2}$.

The annual leakage loss of 1T-DCPV-18/ $\theta_{e}$, contributed by sky diffuse radiation $\left(S_{L, d}\right)$, is presented in Figure 15 in terms of $\mathrm{S}_{\mathrm{L}, \mathrm{d}} / \mathrm{S}_{\mathrm{L}}$. Obviously, almost all leakage loss comes from sky diffuse radiation as $\theta_{e}<83.6^{\circ}$, whereas for $\theta_{e}=90^{\circ}$, about $75 \%$ of leakage loss is attributed to direct sunlight. This implies that DCPC- $18 / \theta_{e}$ with $\theta_{e}<90^{\circ}$ can avoid leakage loss of direct sunlight but can't for sky diffuse radiation. 


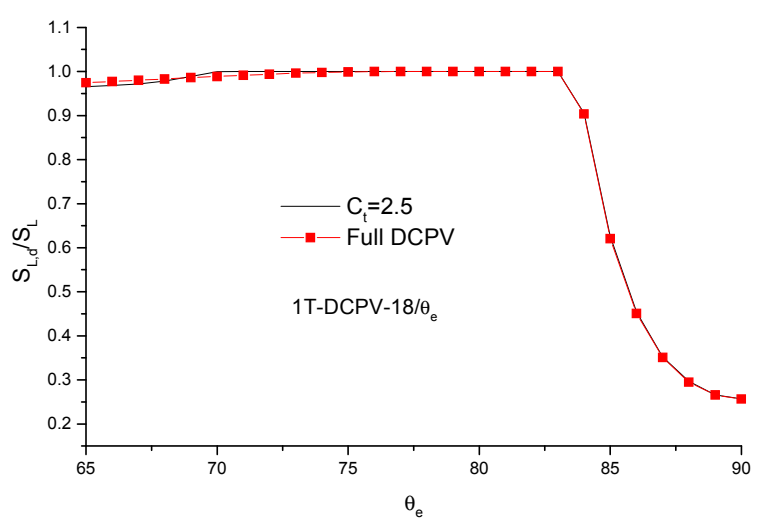

Figure 15. Effects of $\theta_{e}$ on annual leakage loss contributed by sky diffuse radiation for 1T-DCPV-18/ $\theta_{e}$.

Figure 16 shows that, regardless of $C_{t}$, with the increase of $\theta_{e}$, the annual leakage loss of 1T-DCPV-18 $/ \theta_{e}$ decreases when $\theta_{e}<83.6^{\circ}$ and then increases when $\theta_{e}>83.6^{\circ}$, and the optimal $\theta_{e}$ for minimizing annual leakage loss is $\theta_{e, o p t}=83.6^{\circ}$. Effects of $\theta_{e}$ on solar leakage and power loss of 2T-DCPV-12/ $\theta_{e}$ and 3T-DCPV-18/ $\theta_{e}$ are presented in Figures 17 and 18 . It is seen that the annual leakage loss is sensitive to $\theta_{e}$, the optimal $\theta_{e}$ of 2T-DCPV-12/ $\theta_{e}$ for minimizing leakage loss is about $85^{\circ}$, whereas for 3T-DCPV- $18 / \theta_{e}$, the optimal $\theta_{e}$ is $90^{\circ}$. Figures $16-18$ also show that the ratio of annual leakage loss to the annual collectible radiation $\left(S_{a}\right)$ is less than $0.7 \%$, and the power loss of DCPVs due to solar leakage is less than $0.15 \%$. These indicate that the power loss due to solar leakage is insignificant.
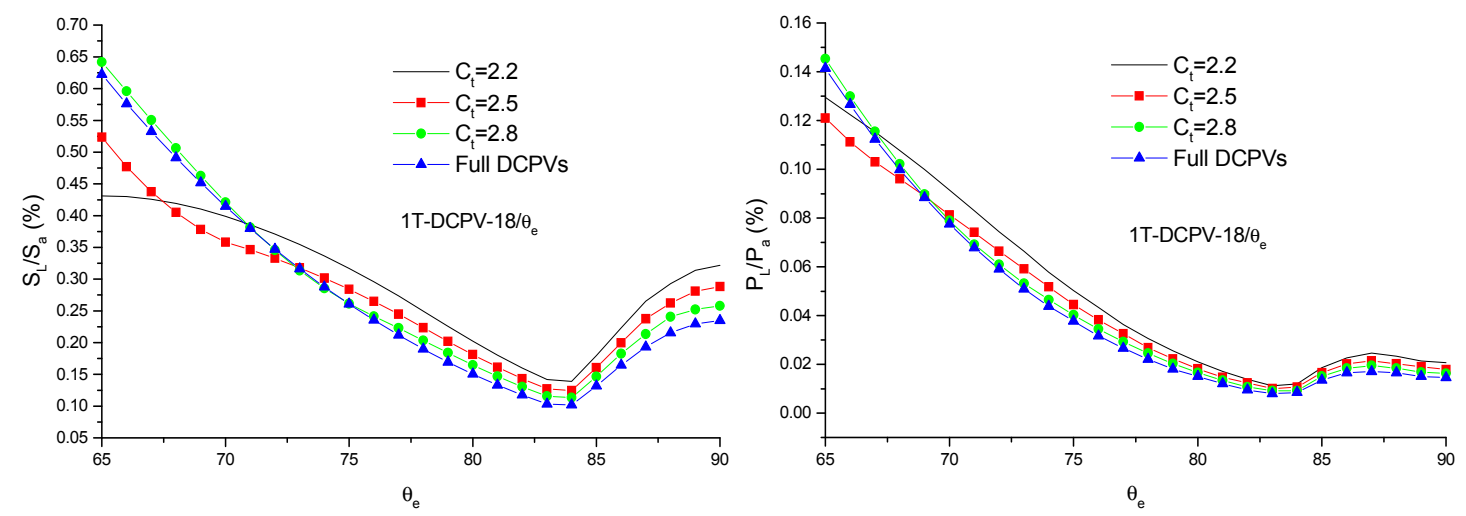

Figure 16. Effects of $\theta_{e}$ on annual radiation and power losses of 1T-DCPV-18/ $\theta_{e}$ due to leakage.
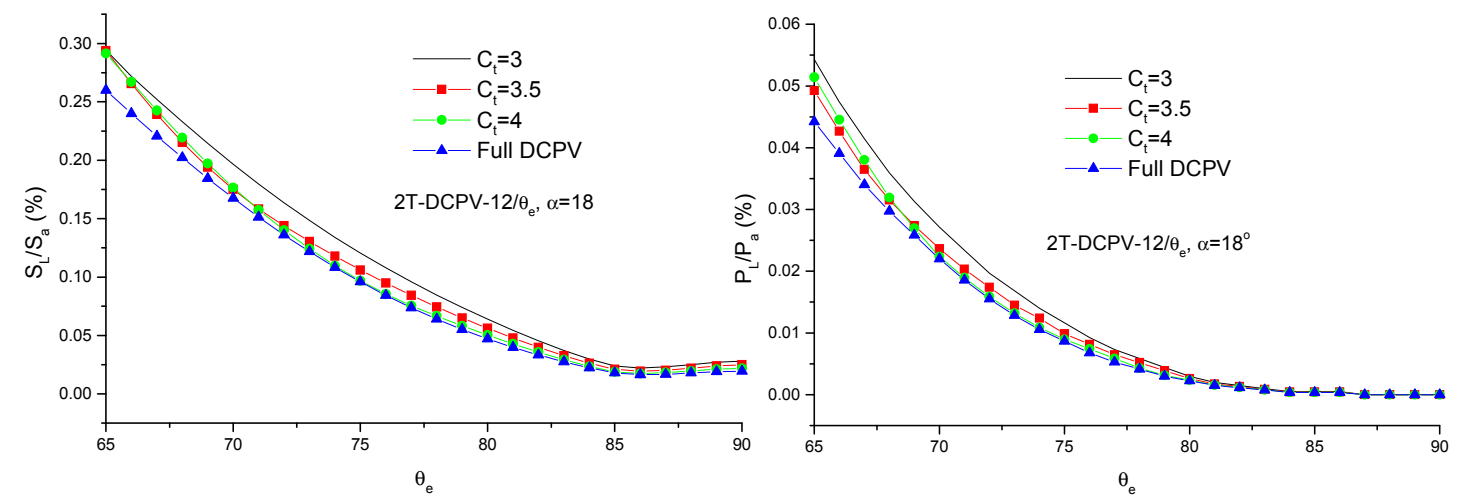

Figure 17. As in Figure 16 but for 2T-DCPV-12/ $\theta_{e}$. 

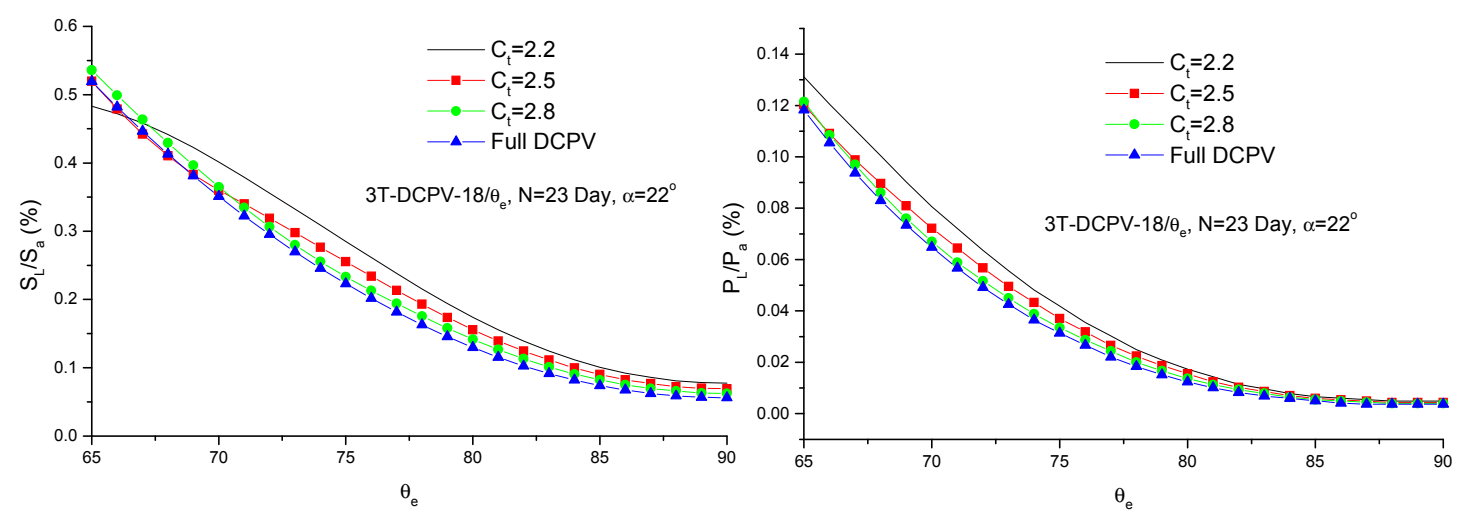

Figure 18. As in Figure 16 but for 3T-DCPV-18/ $\theta_{e}$.

The previous work of the authors [31] indicates that, to make radiation within $\theta_{a}$ totally internally reflected onto solar cells, $\theta_{e}$ should be set to such value making the incidence angle of solar rays on the plane wall $\left(\theta_{\mathrm{i}, \mathrm{pl}}\right)$ larger than $\theta_{\mathrm{c}}$ at solar-noon in solstices, equinoxes and days when tilt-angle adjustment from site latitude is made for 1T, 2T- and 3T-DCPVs, namely, $\theta_{e} \leq \pi+\theta_{a}-2 \theta_{r, 0}-2 \theta_{c}$. However, with the decrease of $\theta_{e}$, more radiation incident on the upper parabolic walls at $\theta_{p}>\theta_{a}$ will be lost by leaking from the opposite plane wall as aforementioned, therefore, the optimal $\theta_{e}$ for minimizing annual leakage loss should be subject to:

$$
\theta_{e, o p t}=\pi+\theta_{a}-2 \theta_{r, 0}-2 \theta_{c}
$$

where $\theta_{r, 0}$ is the refractive angle of incident solar rays at solar-noon, and given by [31]:

$$
\sin \theta_{r, 0}=\left\{\begin{array}{cc}
0.3979 / n & 1 T-D C P V \\
\sin \alpha / n & 2 T-D C P V \\
\sin \left(\alpha-\delta_{N+1}\right) / n & 3 T-D C P V
\end{array}\right.
$$

where $\alpha$ is the tilt-angle adjustment from site latitude for 2T- and 3T-DCPVs, and $\delta_{N=1}$ is the declination of the Sun on days when tilt-angle adjustment from site latitude is made for 3T-DCPVs. It must be noted that, $\theta_{e, o p t}=90^{\circ}$ when $\pi+\theta_{a}-2 \theta_{r, 0}-2 \theta_{c}>0.5 \pi$ because $\theta_{e} \leq 90^{\circ}$. It is known from Equations (86) and (87) that the optimal $\theta_{e}$ are $83.64^{\circ}, 84.62^{\circ}$ and $90^{\circ}$ for 1T-DCPV- $18 / \theta_{e}$, 2T-DCPV- $12 / \theta_{e}\left(\alpha=18^{\circ}\right)$ and 3T-DCPV- $18 / \theta_{e}\left(\alpha=22^{\circ}, \mathrm{N}=23, \delta_{N+1}=9.07^{\circ}\right)$, respectively, in highly agreement with those aforementioned.

\subsection{Optical Performance of DCPVs}

Time variations of optical efficiency of full 1T-DCPVs on the summer solstice are shown in Figure 19, and it is seen that, for 1T-DCPV-18/90, the optical efficiency during the period about $2 \mathrm{~h}$ from solar-noon is lower than those of DCPC-18/65 and DCPV-65/80. This is because for 1T-DCPV-18/ $\theta_{e}$ with $n=1.5, \theta_{e}$ should be less than $83.6^{\circ}$ to ensure direct sunlight totally internally reflected onto solar cells at solar-noon during solstices [31]. It is also seen that $f=0$ for DCPV-18/90 but not for DCPV-18/65 and DCPV-18/80 after 15:40 $\left(\theta_{p}>18^{\circ}\right.$ after 15:40), a result of fact that, for DCPV- $\theta_{a} / \theta_{e}$ with $\theta_{e}<90^{\circ}$, a fraction of radiation incident on the plane wall at $\theta_{p}>\theta_{a}$ arrives on solar cells. 


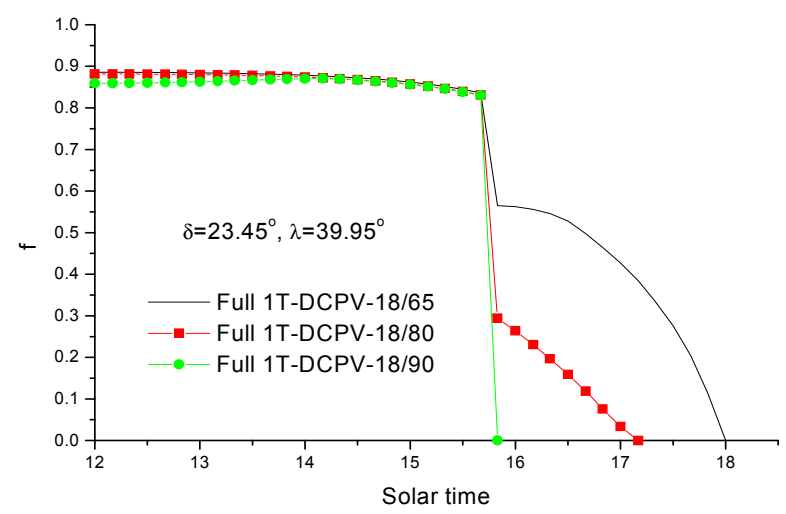

Figure 19. Time variations of optical efficiency of full 1T-DCPVs in summer solstice.

Figure 20 presents effects of $\theta_{e}$ on the annual average optical efficiency $\left(f_{a}\right)$ and annual collectible radiation (in terms of $C_{s}$ ) of 1T-DCPV-18/ $\theta_{e}$. It is seen that, for full 1T-DCPV-18/ $\theta_{e}, f_{a}$ decreases with the increase of $\theta_{e}$, a result of the fact that, with the increase of $\theta_{e}$, the height of DCPVs increases hence radiation loss due to solar absorption increases. Figure 20 also shows that, given $C_{t}$, for slightly truncated DCPVs (i.e., with a large $C_{t}$ ), $f_{a}$ slightly increases with the increase of $\theta_{e}$, whereas for highly truncated DCPVs, it slightly decreases or almost remains unchanged. This is because, for DCPVs with given $\theta_{a}$ and $C_{t}$, with the increase of $\theta_{e}$, the height decreases thus optical efficiency for slightly truncated DCPVs increases due to reduced solar absorption; whereas for highly truncated DCPVs, $h$ is weakly sensitive to $\theta_{e}$, but in another hand, the edge-ray angle $\left(\theta_{t}\right)$ increases with $\theta_{e}$, thus more radiation incident on the parabolic walls at $\theta_{a}<\theta_{p}<\theta_{t}$ is rejected. It is seen from Figure 20 that, given $\theta_{a}$ and $\theta_{e}$, $f_{a}$ decreases with the increase of $C_{t}$ due to increased solar absorption. It is also seen from Figure 20 (right) that, for truncated DCPVs with a given $C_{t}$, the variation trends of annual collectible radiation in terms of $C_{s}$ are identical to those of $f_{a}$ because $C_{s}=f_{a} C_{t}$; whereas for full DCPVs, with increase of $\theta_{e}, C_{s}$ increases due to increased geometric concentration although $f_{a}$ decreases. A similar situation is also observed for 3T-DCPVs as shown in Figure 21, and it indicates that the annual solar gains of full DCPVs are even lower than those of truncated DCPV due to high solar absorption of full DCPVs. These results indicate that, with the increase of $\theta_{e}$, the annual collectible radiation increases for full and slightly truncated DCPVs, thus DCPV- $\theta_{a} / 90$ is favorable from the point of annual radiation collection; whereas for highly truncated DCPVs with a given $C_{t}$, it is almost kept unchanged, thus, to save dielectric material, DCPV- $\theta_{a} / 90$ are advisable.
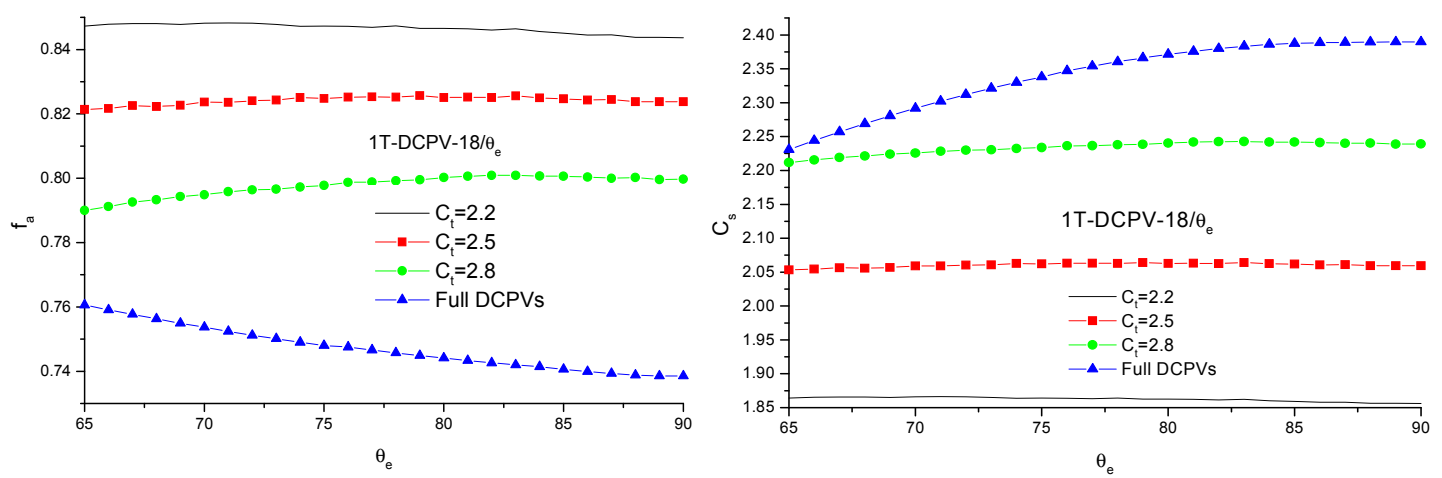

Figure 20. Effects of $\theta_{e}$ on the annual average optical efficiency (Left) and collectible radiation (Right) of 1T-DCPV-18/ $\theta_{e}$. 

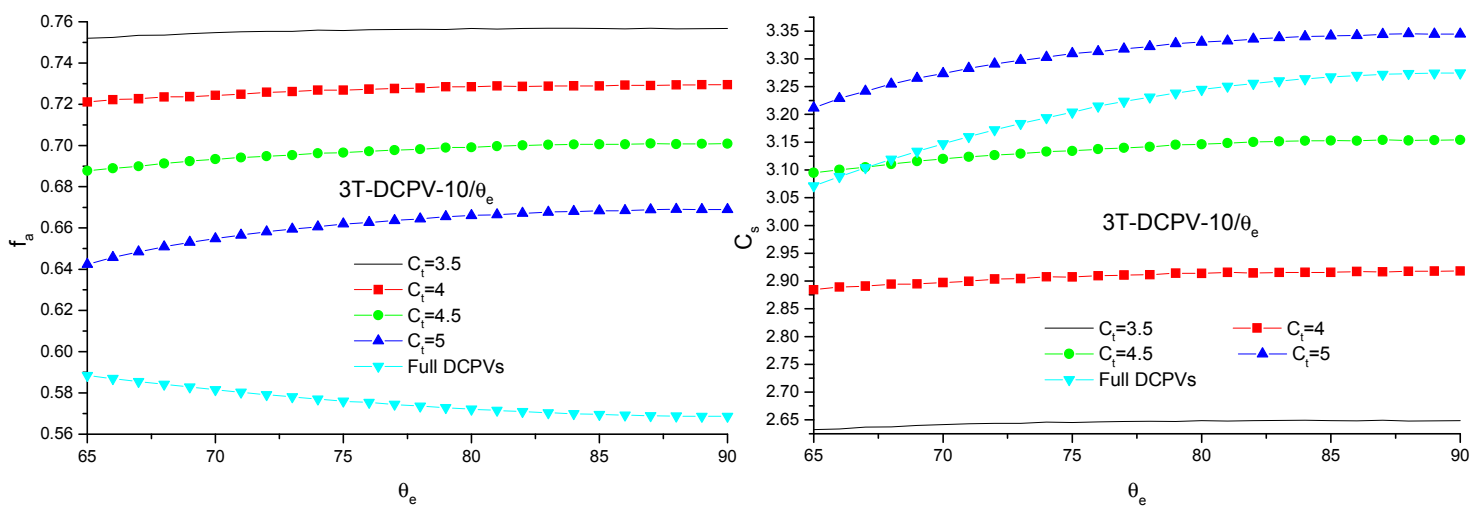

Figure 21. As in Figure 20 but for 3T-DCPV-10/ $\theta_{e}$.

Effects of tilt-angle adjustment strategy on the annual average optical efficiency of DCPV- $18 / \theta_{e}$ is presented in Figure 22, and shows that the $f_{a}$ of 2T- and 3T-DCPVs are almost identical but obviously higher than those of 1T-DCPVs. This means that, periodical tilt-angle adjustment facilitates improving the performance of DCPVs but more frequent adjustment is not advisable. Figure 23 shows effects of solar cells' size on the performance of DCPVs. As expected, increase the size of solar cells results in decrease of $f_{a}$ due to increased path length of solar rays, but it has not effect on annual average photovoltaic efficiency of solar cells $\left(C_{p v}=\eta_{a} / \eta_{a, 0}\right)$ because $\eta_{a}$ is sensitive to angular distribution of annual collectible radiation, and the angular distribution of $S_{a}$ is not sensitive to solar cells' size.
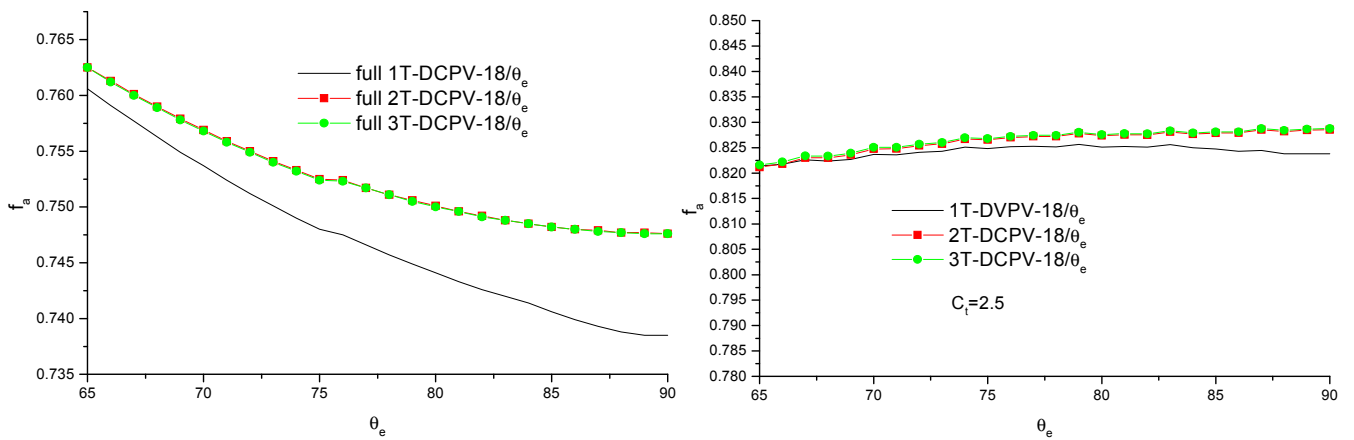

Figure 22. Effects of tilt-angle adjustment strategy on the average annual optical efficiency of DCPV-18/ $\theta_{e}$.

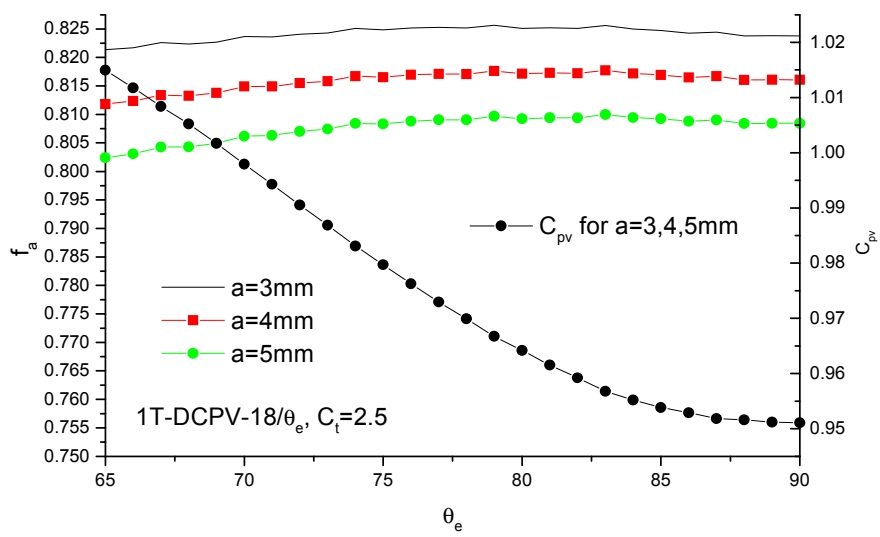

Figure 23. Effects of solar cells' size on the performance of 1T-DCPV-18/ $\theta_{e}$.

\subsection{Photovoltaic Performance of DCPVs}

Figure 24 presents effects of $\theta_{e}$ on the annual average photovoltaic efficiency of solar cells in terms of $C_{p v}$. It is seen that $C_{p v}$ is sensitive to the geometry of DCPV $\left(\theta_{a}, \theta_{e}\right.$ and $\left.C_{t}\right)$ and tilt-angle adjustment 
strategy, but not to the size of solar cells (see Figure 23) and the extinction coefficient of the dielectric $(k)$ because solar absorption has effects on solar gain but not on the angular distribution of solar gain. As shown in Figure 24, regardless of whether full or truncated DCPVs are considered $C_{p v}$ always decreases with the increase of $\theta_{e}$ because, with the increase of $\theta_{e}$, more radiation arrives on solar cells at large angles [29], and given $\theta_{e}, C_{p v}$ increases with $C_{t}$ as the incident angle $\left(\theta_{i n}\right)$ of radiation reflecting from upper parabolic walls is small. It is found from Figure 24 that $C_{p v}$ is even higher than 1 for $\theta_{e}<90^{\circ}$; whereas for DCPVs with $\theta_{e}=90^{\circ}, C_{p v}$ is always less than 1 but higher than 0.935 . This is because, as compared to similar non-concentrating PV panel, for DCPVs with $\theta_{e}=65^{\circ}$, more radiation annually arrives solar cells at $\theta_{i n}<60^{\circ}$ thanks to refraction of incident rays at the aperture as seen from Figure 25; whereas for DCPVs with $\theta_{e}=90^{\circ}$, more radiation arrives on solar cells at $\theta_{i n}>60^{\circ}$. These results indicate that, the electric loss of DCPVs due to increased incident angle on solar cells after radiation concentration is considerably small, and the annual average photovoltaic efficiency $\left(\eta_{a}\right)$ of solar cells for radiation concentrated by DCPC $-\theta_{a} / \theta_{e}$ with $\theta_{e}<90^{\circ}$ is even higher than that of similar non-concentrating PV panel $\left(\eta_{a, 0}\right)$.
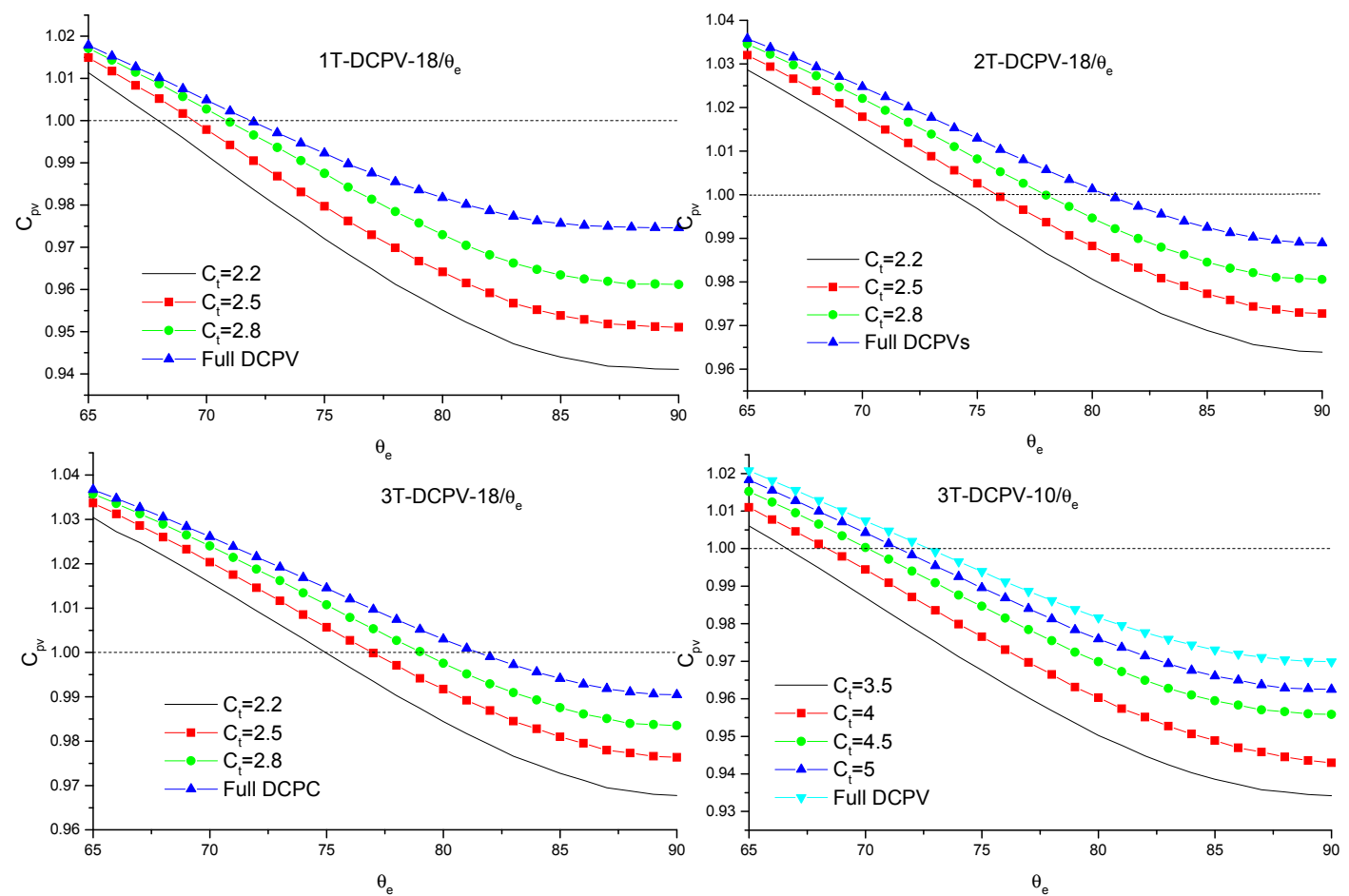

Figure 24. Effects of $\theta_{e}$ on the annual average photovoltaic efficiency of solar cells. 

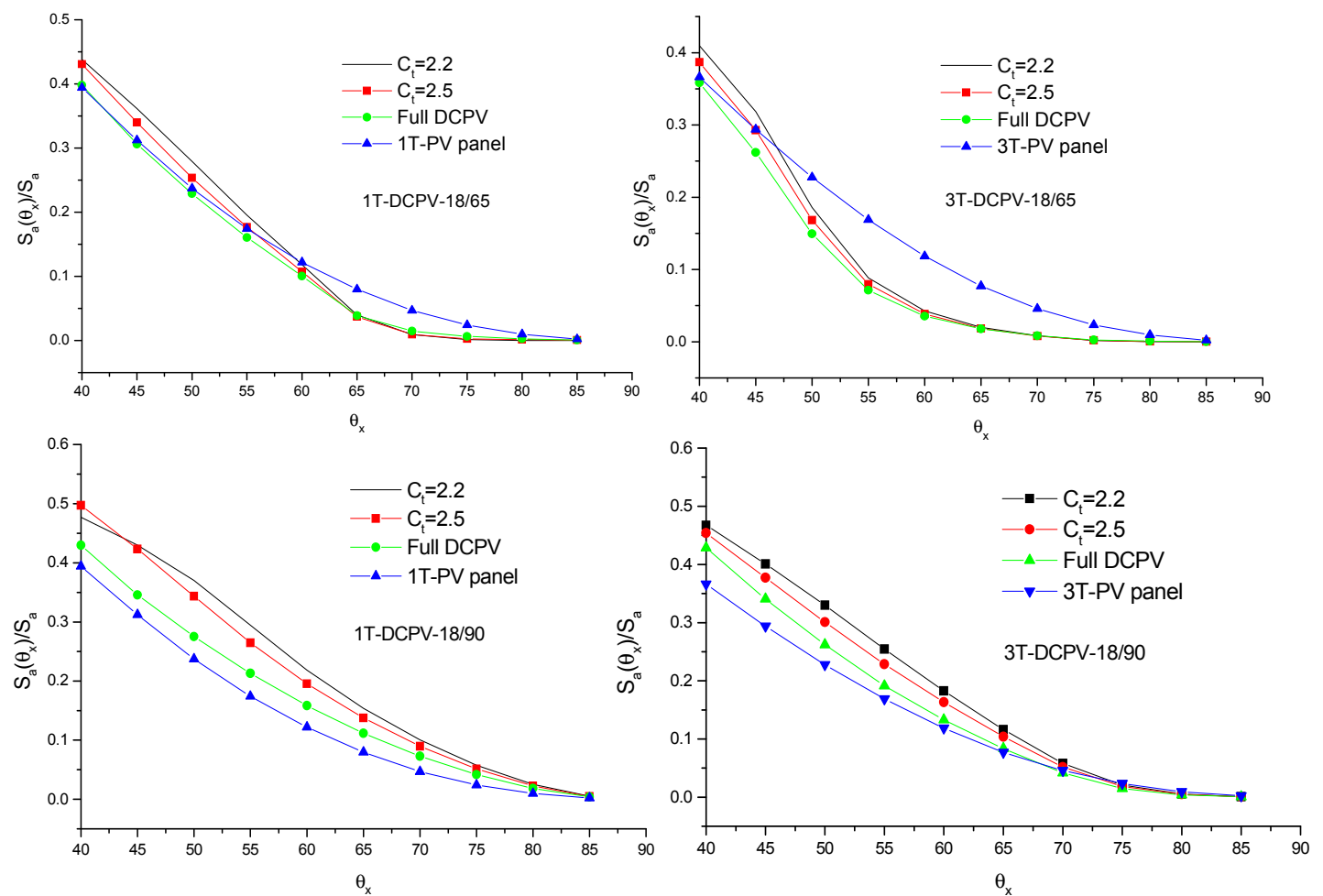

Figure 25. Angular distribution of annual collectible radiation on solar cells in terms of $S_{a}\left(\theta_{x}\right) / S_{a}$.

Effects of geometry of DCPC on $f_{a} C_{p v}$, the photovoltaic performance coefficient of DCPVs as compared to similar non-concentrating PV panel, are presented in Figure 26. It shows that $f_{a} C_{p v}$ always decreases with the increase of $\theta_{e}$ except for slightly truncated 3T-DCPV-10/ $\theta_{e}\left(C_{t}=5\right)$ where $f_{a} C_{p v}$ slightly increase for $\theta_{e}<69^{\circ}$, and this implies that, the use of DCPC with a restricted exit angle facilities improving performance of DCPVs, thus, DCPV- $\theta_{a} / \theta_{e}$ with $\theta_{e}<90^{\circ}$ is favorable.
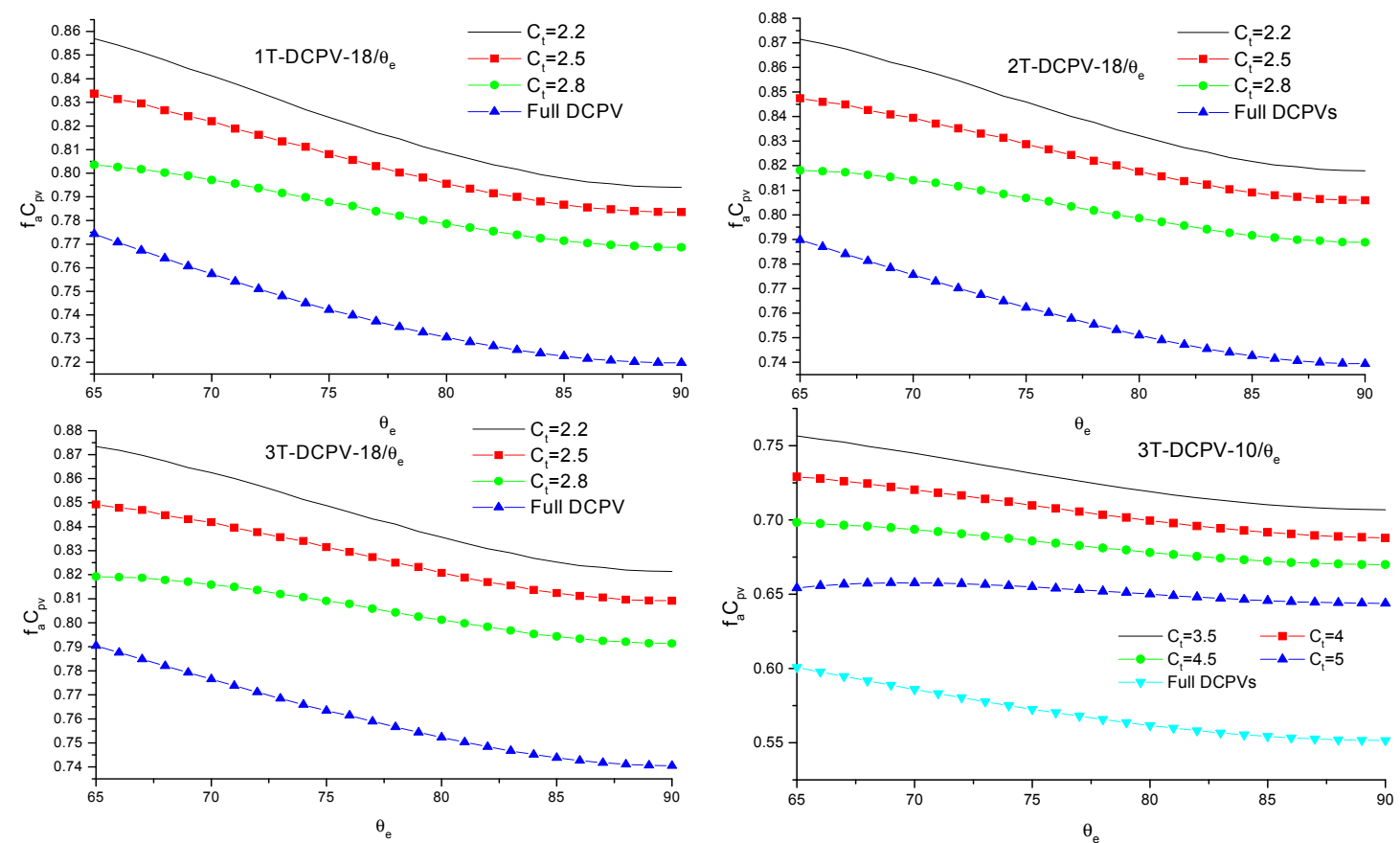

Figure 26. Effects of $\theta_{e}$ on $f_{a} C_{p v}$, the photovoltaic performance coefficient of DCPVs. 
Figure 27 presents effects of $\theta_{e}$ on annual power output of full DCPVs in terms of $C_{p}$, and it is seen that, for full DCPVs with a given $\theta_{a}$, the annual power output increases with the increase of $\theta_{e}$ due to increased geometric concentration. As aforementioned, $C_{p}=f_{a} C_{p v} C_{t}$, therefore, for truncated DCPVs with a given $\theta_{a}$ and $C_{t}, C_{p}$ decreases with the increase of $\theta_{e}$ because $f_{a} C_{p v}$ decreases with $\theta_{e}$. This implies that, from the point of annual electricity generation, DCPV- $\theta_{a} / 90$ are favorable for full DCPVs, but for truncated DCPVs with given $\theta_{a}$ and $C_{t}$, DCPVs with $\theta_{e}<90^{\circ}$ are favorable.

It is observed from Figure 27 that, the $C_{p}$ of full 2T-and 3T-DCPV-18/ $\theta_{e}$ are almost identical, and compared to 1 T-DCPC- $18 / \theta_{e}, C_{p}$ of 2 T- and 3T-DCPV- $18 / \theta_{e}$ is about $7-8 \%$ and $8-9 \%$ higher, respectively. This indicates that the periodical tilt-angle adjustment can improve performance of DCPVs, but more frequent tilt-angle adjustment is not advisable.

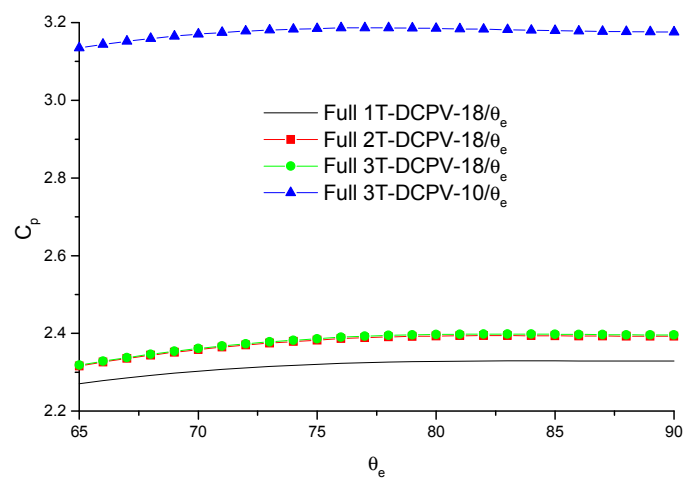

Figure 27. Effects of $\theta_{e}$ on annual power output increase factor of full DCPVs.

To evaluate the contribution per unit volume of dielectric material to the annual electricity generation, the ratio of annual power output $\left(P_{a}\right)$ to the volume of dielectric material used for constructing DCPCs, $P_{a, v}$, is introduced. As shown in Figure 28, with the increase of $\theta_{e}, P_{a, v}$ decreases for full DCPVs and increases for truncated DCPVs. This implies that, from the point of contribution per unit volume dielectric to the annual electricity generation, DCPVs with $\theta_{e}<90^{\circ}$ are favorable for full DCPVs, and for truncated DCPVs, DCPV- $\theta_{a} / 90$ are favorable. Results in Figure 28 also indicate that, with the decrease of $C_{t}, P_{a, v}$ increases greatly, a result of the fact that the contribution of upper parabolic walls of DCPC to the radiation concentration is limited [51]. As an example, for DCPC-18/90, when $\theta_{t}$ varies from $\theta_{a}\left(18^{\circ}\right)$ to $34^{\circ}, C_{t}$ slightly decreases from 3.24 to 2.8 , but the cross-section area $\left(\mathrm{A}_{\mathrm{c}}\right)$ sharply decreases from 17.52 to 6.04 , and $P_{a, v}$ of $1 \mathrm{~T}-\mathrm{DCPV}-18 / 90$ greatly increases from $3794.7 \mathrm{MJ} / \mathrm{m}^{3}$ to $10324.3 \mathrm{MJ} / \mathrm{m}^{3}$, increasing by a factor of 2.72 times. This indicates that, in practical applications, truncated DCPVs should be employed to increase $P_{a, v}$ and save dielectric material, thus DCPV- $\theta_{a} / 90$ are advisable. 

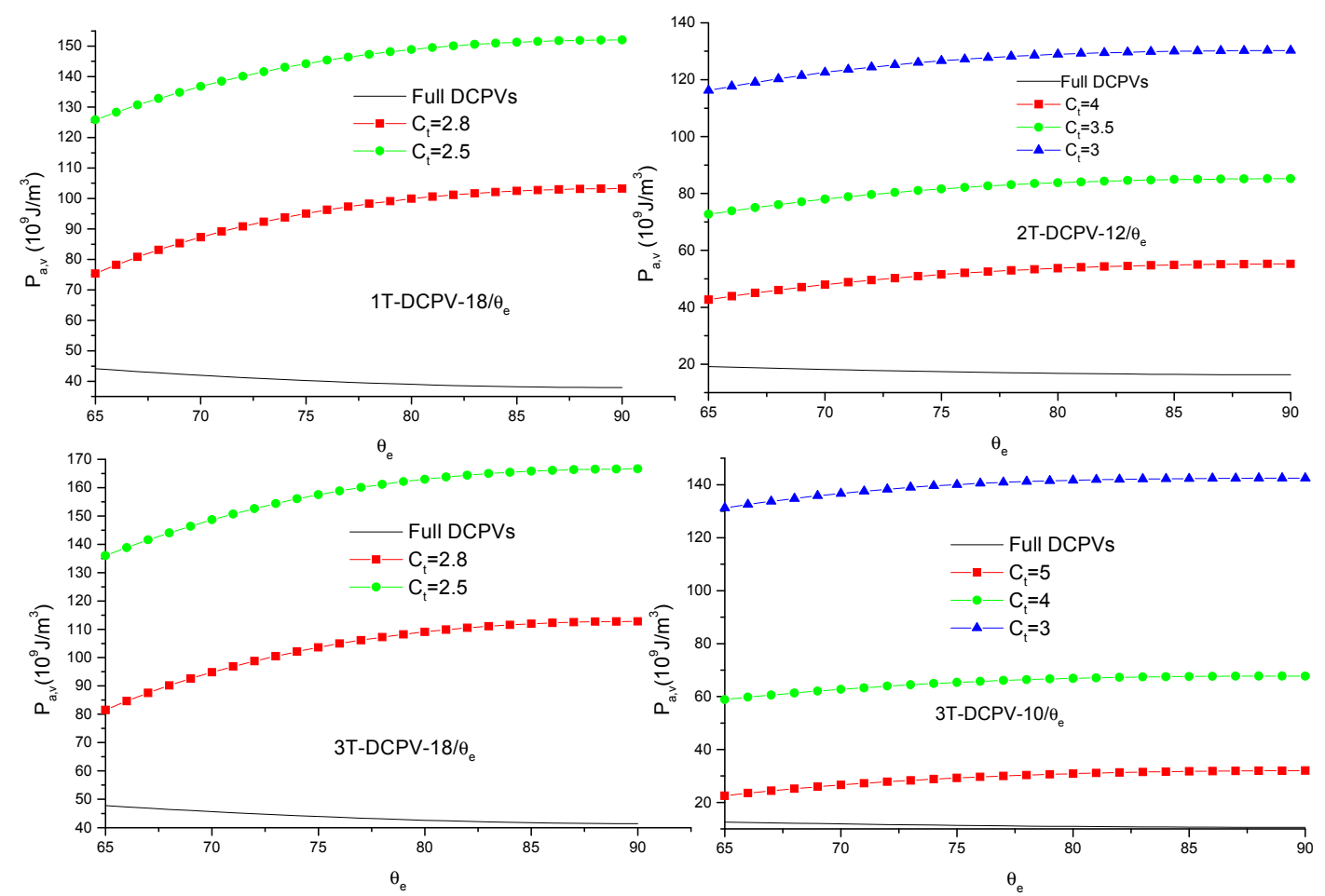

Figure 28. Effects of $\theta_{e}$ on contribution per unit volume of dielectric to annual power output of DCPVs.

\section{Conclusions}

To investigate the performance of DCPV- $\theta_{a} / \theta_{e}$, a detailed mathematical procedure is suggested based on three-dimensional radiation transfer model and angular dependence of photovoltaic efficiency of solar cells. This model allows one to reasonably predict the optical performance of DCPCs and evaluate the effects of a DCPV's geometry on its optical and photovoltaic performance, thus being helpful for the design of DCPVs. Although the model can't reasonably predict the photovoltaic performance of DCPVs, this would not affect results of comparative study on performance of DCPV with different geometry because all theoretical results are obtained under the assumption that, except for incidence angle, the effects of all factors on the photovoltaic performance of DCPVs are identical.

Analysis shows that, the use of DCPC with a restricted exit angle can avoid leakage of radiation irradiating on the lower parabolic walls for $\theta_{p} \leq \theta_{a}$, but it results in a leakage loss of radiation irradiating on the upper parabolic walls at $\theta_{p}>\theta_{a}$ first and then leaking from the opposite plane wall. Calculations show that, the annual radiation leakage is sensitive to the geometry of DCPC and number of periodical tilt-angle adjustments in a year, and the optimal $\theta_{\text {e.opt }}$ for minimizing annual leakage is the one that makes the incidence angle of solar rays on the plane wall equal to $\theta_{c}$ at solar-noon on solstices, equinoxes and days when a tilt-angle adjustment from site latitude is made for 1T, 2T- and 3T-DCPVs, respectively. It is found that annual radiation leakage is considerable small, for DCPVs with $\theta_{e}<\theta_{e . o p t}$, almost all leaked radiation comes from sky diffuse radiation, whereas for $\theta_{e}=90^{\circ}$, most of leakage loss is attributed to direct sunlight.

Results show that, for full and slightly truncated DCPVs, the annual collectible radiation increase with the increase of $\theta_{e}$; whereas for highly truncated DCPVs with a given $\theta_{a}$ and $C_{t}$, it almost remains unchanged.

Analysis and calculations indicate that the annual average photovoltaic efficiency of solar cells for concentrated radiation is sensitive to the geometry of DCPV- $\theta_{a} / \theta_{e}$ and tilt-angle adjustment strategy in a year but not to solar cells' size and extinction coefficient of the dielectric. As compared to similar non-concentrating PV panels, more radiation annually arrives on solar cells of DCPV- $\theta_{a} / \theta_{e}$ at small 
angles thanks to refraction of radiation on the aperture, hence, under same operation condition, the annual average photovoltaic efficiency of solar cells is even higher.

Analysis also shows that, the power increase factor of DCPVs being much less than the geometric concentration of DCPCs is mainly attributable to optical losses due to absorption of solar rays on their way to solar cells, and power loss due to radiation leakage and increased incident angle of solar rays on solar cells after concentration is not significant.

From the point of view of annual electricity generation, DCPV- $\theta_{a} / 90$ are favorable for full DCPVs with a given $\theta_{a}$, and for truncated DCPVs with a given $\theta_{a}$ and $C_{t}$, DCPVs with $\theta_{e}<90^{\circ}$ are favorable; whereas from the point of contribution per unit volume of dielectric to the annual electricity generation, the situation is reversed. In practical applications, truncated DCPVs are recommended to save dielectric and reduce solar absorption of dielectric, thus DCPV- $\theta_{a} / 90$ are advisable.

To reduce power losses due to dielectric solar absorption, DCPCs are suitable for use to concentrate radiation on small size of solar cells. To improve the performance of DCPVs, yearly adjusting tilt-angle of DCPVs' aperture two or four times is advisable, but more frequent adjustment is not advisable.

Author Contributions: R.T. the sponsor of the work; G.L. responsible for the development of mathematical model and result analysis; J.T. student for Master program, is responsible for the numerical calculations.

Funding: This work is partial fulfillment of funded program 51466016, financially supported by Nature Science Foundation of China.

Conflicts of Interest: The authors declare no conflict of interest.

\section{Glossary}

a width of solar cells in DCPVs (m)

$A_{c} \quad$ area of cross-section of DCVs $\left(\mathrm{m}^{2}\right)$

$C_{t} \quad$ geometric concentration of DCPVs (dimensionless)

$C_{p v} \quad$ ratio of annual average photovoltaic efficiency of solar cells for concentrated radiation to that of

similar non-concentrating solar cells (dimensionless)

$C_{p} \quad$ annual power output increase factor of DCPVs as compared to similar PV panel (dimensionless)

$C_{S} \quad$ annual solar gain increase factor of DCPVs as compared to similar PV panel (dimensionless)

$f \quad$ optical efficiency of DCPVs (dimensionless)

$f_{a} \quad$ annual average optical efficiency of DCPVs (dimensionless)

$H \quad$ daily radiation $\left(\mathrm{J} / \mathrm{m}^{2}\right)$

$h \quad$ height of DCPVs (m)

I collectible radiation or radiation intensity $\left(\mathrm{W} / \mathrm{m}^{2}\right)$

$k \quad$ extinction coefficient of dielectric material $(1 / \mathrm{m})$

$L \quad$ path length of solar rays from the aperture to solar cells of DCPVs (m)

$N \quad$ Day number counting from equinoxes

$n \quad$ refractive index of dielectric (dimensionless)

$n_{M} \quad$ unit vector of the normal to parabolic wall at point $\mathrm{M}$

$n_{S} \quad$ unit vector of incident solar rays

$n_{r} \quad$ unit vector of refractive solar rays

$r_{M} \quad$ unit vector of solar ray reflecting from $\mathrm{M}$ on parabolic wall

$P_{a} \quad$ annual power output from DCPVs $\left(\mathrm{MJ} / \mathrm{m}^{2}\right)$

$P_{a, v} \quad$ annual power output per unit volume of dielectric from DCPVs $\left(\mathrm{J} / \mathrm{m}^{3}\right)$

$P_{0} \quad$ annual power output from similar PV panel $\left(\mathrm{MJ} / \mathrm{m}^{2}\right)$

$\mathrm{S}_{\mathrm{a}} \quad$ annual collectible radiation on solar cells of DCPVs $\left(\mathrm{MJ} / \mathrm{m}^{2}\right)$

$\mathrm{S}_{\mathrm{a}}\left(\theta_{x}\right) \quad$ annual collectible radiation on solar cells with $\theta_{\text {in }} \geq \theta_{x}\left(\mathrm{MJ} / \mathrm{m}^{2}\right)$

$S_{0} \quad$ annual collectible radiation on aperture of DCPVs or similar PV panel (MJ/m2)

$S_{L} \quad$ annual radiation leakage of DCPVs $(\mathrm{MJ} / \mathrm{m} 2)$

$T \quad$ solar time (s)

$x, z \quad x$-and $z$-coordinates of any point on walls of DCPC (m) 


\section{Greek letters}

$\alpha$ : tilt-angle adjustment of DCPVs' aperture from site latitude (the unit of all angles is radians in

mathematical expressions and degrees in the text)

$\beta \quad$ tilt-angle of DCPVs' aperture relative to horizon

$\delta \quad$ declination of the Sun

$\delta_{N+1}$ declination of the Sun in the days when tilt-angle adjustment is made for 3T-DCPVs

$\varphi \quad$ polar angle of any point on parabolic wall

$\eta \quad$ photovoltaic conversion efficiency of DCPVs

$\eta_{p v} \quad$ photovoltaic efficiency of solar cells (dimensionless)

$\gamma \quad$ tilt-angle of any line relative to $\mathrm{x}$-axis

$\lambda \quad$ site latitude

$\theta_{a} \quad$ acceptance half-angle of DCPCs

$\theta_{c} \quad$ critical incident angle for total internal reflection at dielectric-air interface

$\theta_{e} \quad$ maximum exit angle of DCPC-/for refractive radiation within

$\theta_{a p} \quad$ incident angle of solar rays on the aperture of DCPCs

$\theta_{\text {in }} \quad$ incident angle of solar rays on solar cells

$\theta_{i, M} \quad$ incident angle of solar ray at point $\mathrm{M}$ of parabolic wall of DCPCs

$\theta_{r} \quad$ Refractive angle of incident solar rays on the aperture

$\theta_{r, 0}: \quad$ Refractive angle of incident solar rays at solar-noon

$\theta_{r, M} \quad$ exit angle of solar ray reflecting from $\mathrm{M}$

$\theta_{p} \quad$ projected angle of refractive solar rays on the cross-section of DCPV

$\theta_{t} \quad$ edge-ray angle of truncated DCPCs

$\rho \quad$ reflectivity of solar ray on walls of DCPCs (dimensionless)

$\psi \quad$ opening angle of $\mathrm{V}$ concentrator formed by two plane walls of DCPCs

$\tau \quad$ transmittance of sola ray on the aperture (dimensionless)

$\tau \quad$ transmittance of solar ray from aperture to solar cells due to solar absorption (dimensionless)

$\omega \quad$ hour angle

\section{Subscripts}

$\begin{array}{ll}a b s & \text { absorber of DCPC } \\ a p & \text { aperture } \\ b & \text { beam radiation } \\ c & \text { critical value } \\ d & \text { sky diffuse radiation } \\ i & \text { incident solar ray } \\ L & \text { radiation loss due to leakage } \\ r & \text { refractive ray; } \\ p l & \text { plane wall }\end{array}$

\section{References}

1. Koussa, M.; Cheknane, A.; Hadji, S.; Haddadi, M.; Noureddine, S. Measured and modelled improvement in solar energy yield from flat plate photovoltaic systems utilizing different tracking systems and under a range of environmental conditions. Appl. Energy 2011, 88, 1756-1771. [CrossRef]

2. Li, Z.; Liu, X.; Tang, R. Optical performance of inclined south-north single-axis tracked solar panels. Energy 2010, 35, 2511-2516. [CrossRef]

3. Jaaz, A.H.; Hassan, H.A.; Sopian, K.; Ruslan, M.H.B.H.; Zaidi, H. Design and development of compound concentrating for photovoltaic solar collector: Review. Renew. Sustain. Energy Rev. 2017, 76, 1108-1121. [CrossRef]

4. Chong, K.K.; Lao, S.L.; Yew, T.K.; Tan, P.C.L. Design and development in optics of concentrator photovoltaic system. Renew. Sustain. Energy Rev. 2013, 19, 598-612. [CrossRef]

5. Mallick, T.K.; Eames, P.C.; Hyde, T.J.; Norton, B. The design and experimental characterization of an asymmetric compound parabolic photovoltaic concentrator for building façade integration in the UK. Sol. Energy 2004, 77, 319-327. [CrossRef] 
6. Mallick, T.K.; Eames, P.C.; Norton, B. Non-concentrating and asymmetric compound parabolic concentrating building façade integrated photovoltaic: An experimental comparison. Sol. Energy 2006, 80, 834-849. [CrossRef]

7. Mallick, T.K.; Eames, P.C. Design and fabrication of low concentrating second generation PRIDE concentrator. Sol. Energy Mater. Sol. Cells 2007, 91, 597-608. [CrossRef]

8. Brogren, M.; Wennerberg, J.; Kapper, R.; Karlsson, B. Design of concentrating elements with CIS thin-film solar cells for façade integration. Sol. Energy Mater. Sol. Cells 2003, 75, 567-575. [CrossRef]

9. Yousef, M.S.; Rahman, A.K.A.; Ookawara, S. Performance investigation of low-Concentration photovoltaic systems under hot and arid conditions: Experimental and numerical results. Energy Convers. Manag. 2016, 128, 82-94. [CrossRef]

10. Tang, R.; Wang, J. A note on multiple reflections of radiation within CPCs and its effect on calculations of energy collection. Renew. Energy 2013, 57, 490-496. [CrossRef]

11. Royne, A.; Dey, C.R.; Mills, D.R. Cooling of photovoltaic cells under concentrated illumination: A critical review. Sol. Energy Mater. Sol. Cells 2005, 86, 451-483. [CrossRef]

12. Yu, Y.; Tang, R.; Xia, C. Irradiation distribution on solar cells inside CPCs with a restricted exit angle. Adv. Mater. Res. 2012, 588, 603-607. [CrossRef]

13. Tang, J.; Yu, Y.; Tang, R. A three-dimensional radiation transfer model to evaluate performance of compound parabolic concentrator-based photovoltaic systems. Energies 2018, 11, 896. [CrossRef]

14. Baig, H.; Heasman, K.C.; Mallick, T.K. Non-uniform illumination in concentrating solar cells. Renew. Sustain. Energy Rev. 2012, 16, 5890-5909. [CrossRef]

15. Hatwaambo, S.; Hakansson, H.; Nilsson, J.; Karlsson, B. Angular characterization of low concentrating PV-CPC using low-cost reflectors. Sol. Energy Mater. Sol. Cells 2008, 92, 1347-1351. [CrossRef]

16. Hatwaambo, S.; Hakansson, H.; Roos, A.; Karlsson, B. Mitigating the non-uniform illumination in low concentrating CPCs using structured reflectors. Sol. Energy Mater. Sol. Cells 2009, 93, 2020-2024. [CrossRef]

17. Yu, Y.; Tang, R. Diffuse reflections within CPCs and its effect on energy collection. Sol. Energy 2015, 120, 44-54. [CrossRef]

18. Tang, F.; Li, G.; Tang, R. Design and optical performance of CPC based compound plane concentrators. Renew. Energy 2016, 95, 140-151. [CrossRef]

19. Baig, H.; Sarmah, N.; Chemisana, D.; Rosell, J.; Mallick, T.K. Enhancing performance of a linear dielectric based concentrating photovoltaic system using a reflective film along the edgy. Energy 2014, 73, 177-191. [CrossRef]

20. Yu, Y.; Liu, N.; Li, G.; Tang, R. Performance comparison of CPCs with and without exit angle restriction for concentrating radiation on solar cells. Appl. Energy 2015, 155, 284-293. [CrossRef]

21. Bahaidarah, H.M.; Gandhidasan, P.; Baloch, A.A.B.; Tanweer, B.; Mahmood, M. A comparative study on the effect of glazing and cooling for compound parabolic concentrator PV systems: Experimental and analytical investigations. Energy Convers. Manag. 2016, 129, 227-239. [CrossRef]

22. Bahaidarah, H.M.; Tanweer, B.; Gandhidasan, P.; Ibrahim, N.; Rehman, S. Experimental and numerical study on non-concentrating and symmetric unglazed compound parabolic photovoltaic concentration systems. Appl. Energy 2014, 136, 527-536. [CrossRef]

23. Brogren, M.; Nostell, P.E.R.; Karlsson, B. Optical efficiency of a PV-thermal hybrid CPC module for high latitudes. Sol. Energy 2001, 69, 173-185. [CrossRef]

24. Elsafi, A.M.; Gandhidasan, P. Comparative study of double-pass flat and compound parabolic concentrated photovoltaic-thermal systems with and without fins. Energy Convers. Manag. 2015, 98, 59-68. [CrossRef]

25. Baig, H.; Siviter, J.; Li, W.; Paul, M.C.; Montecucco, A.; Rollley, M.H.; Sweet, T.K.N.; Gao, M.; Mullen, P.A.; Fernandez, E.F.; et al. Conceptual design and performance evaluate of a hybrid concentrating photovoltaic system in preparation for energy. Energy 2018, 147, 547-560. [CrossRef]

26. Su, Y.H.; Pei, G.; Riffat, B.; Huang, H.L. A novel lens-walled compound parabolic concentrator for photovoltaic applications. J. Sol. Energy Eng. 2012, 134, 021010. [CrossRef]

27. Li, G.Q.; Pei, G.; Su, Y.H.; Ji, J.; Riffat, R. Experiment and simulation study on the flux distribution of lens-walled compound parabolic concentrator compared with mirror compound parabolic concentrator. Energy 2013, 58, 98-403.

28. Rabl, A.; Winston, R. Ideal concentration for finite sources and restricted exit angle. Appl. Opt. 1976, 15, 2880-2883. [CrossRef] [PubMed] 
29. Yu, M.; Yu, Y.; Tang, R. Angular distribution of annual collectible radiation on solar cells of CPC based photovoltaic systems. Sol. Energy 2016, 135, 827-839. [CrossRef]

30. Winston, R. Dielectric compound parabolic concentrators. Appl. Opt. 1976, 15, 291-292. [CrossRef] [PubMed]

31. Li, G.; Tang, J.; Tang, R. A note on design of linear dielectric compound parabolic concentrators. Sol. Energy 2018, 171, 500-507. [CrossRef]

32. Muhammad-Sukki, F.; Abu-Bakar, S.H.; Ramirez-Iniguez, R.; McMeekin, S.G.; Stewart, B.G.; Munir, A.B.; Yasin, S.H.M.; Rahim, R.A. Performance analysis of a mirror symmetrical dielectric totally internally reflecting concentrator for building integrated photovoltaic systems. Appl. Energy 2013, 111, 288-299. [CrossRef]

33. Sellami, N.; Mallick, T.K. Optical efficiency study of PV crossed compound parabolic concentrator. Appl. Energy 2013, 102, 868-876. [CrossRef]

34. Baig, H.; Sellami, N.; Mallick, T.K. Performance modeling and testing of a building integrated concentrating photovoltaic (BICPV) system. Sol. Energy Mater. Sol. Cells 2015, 134, 29-44. [CrossRef]

35. Zacharopoulos, A.; Eames, P.C.; Mclarnon, D.; Norton, B. Linear dielectric non-imaging concentrating covers for PV integrated building facades. Sol. Energy 2000, 68, 439-452. [CrossRef]

36. Brunotte, M.; Geotzberger, A.; Blieske, U. Two-stage concentrator permitting concentration up to $300 \times$ with one-axis tracking. Sol. Energy 1996, 56, 285-300. [CrossRef]

37. Hein, M.; Dimroth, F.; Siefer, G.; Bett, A.W. Characterisation of a $300 \times$ photovoltaic concentrator system with one-axis tracking. Sol. Energy Mater. Sol. Cells 2003, 75, 277-283. [CrossRef]

38. Pei, G.; Li, G.Q.; Su, Y.H.; Ji, J.; Riffat, R.; Zheng, H.F. Preliminary ray tracing and experimental study on the effect of mirror coating on the optical efficiency of a solid dielectric compound parabolic concentrator. Energies 2012, 5, 3627-3639. [CrossRef]

39. Baig, H.; Sarmah, N.; Heasman, K.C.; Mallick, T.K. Numerical modelling and experimental validation of a low concentrating photovoltaic system. Sol. Energy Mater. Sol. Cells 2013, 113, 201-219. [CrossRef]

40. Rabl, A. Active Solar Collectors and Their Applications; Oxford University Press: Oxford, UK, 1985.

41. Rabl, A. Comparison of solar concentrators. Sol. Energy 1976, 18, 93-111. [CrossRef]

42. Khonkar, H.E.I.; Sayigh, A.A.M. Raytrace for compound parabolic concentrator. Sol. Energy 1994, 5, $376-383$. [CrossRef]

43. Tang, R.; Wu, M.; Yu, Y.; Li, M. Optical performance of fixed east-west aligned CPCs used in China. Renew. Energy 2010, 35, 1837-1841. [CrossRef]

44. Wang, Q.; Wang, J.; Tang, R. Design and optical performance of CPCs with evacuated tube as receivers. Energies 2016, 9, 759. [CrossRef]

45. Yu, X.; Su, Y.H. A discussion of inner south projection angle for performance analysis of dielectric compound parabolic concentrator. Sol. Energy 2015, 113, 101-113. [CrossRef]

46. Yu, Y.; Liu, N.; Tang, R. Optical performance of CPCs for concentrating solar radiation on flat receivers with a restricted incidence angle. Renew. Energy 2014, 62, 679-688. [CrossRef]

47. Li, W.; Pau, M.C.; Sellami, N.; Sweet, T.; Montecucco, A.; Siviter, J.; Baig, H. Six-parameter electrical model for photovoltaic cell/module with compound concentrator. Sol. Energy 2016, 137, 551-563. [CrossRef]

48. Tang, R.; Liu, X. Optical performance and design optimization of V-trough concentrators for photovoltaic applications. Sol. Energy 2011, 85, 2154-2166. [CrossRef]

49. Chen, Z.Y. The Climatic Summarization of Yunnan; Weather Publishing House: Beijing, China, 2001.

50. Collares-Pereira, M.; Rabl, A. The average distribution of solar radiation: correlations between diffuse and hemispherical and between hourly and daily insolation values. Sol. Energy 1979, 22, 155-164. [CrossRef]

51. Carvalho, M.J.; Collares-Pereira, M.; Gordon, J.M.; Rabl, A. Truncation of CPC solar collectors and its effect on energy collection. Sol. Energy 1985, 35, 393-399. [CrossRef]

(C) 2018 by the authors. Licensee MDPI, Basel, Switzerland. This article is an open access article distributed under the terms and conditions of the Creative Commons Attribution (CC BY) license (http:/ / creativecommons.org/licenses/by/4.0/). 